\title{
$K$-THEORY AND STEENROD HOMOLOGY: APPLICATIONS TO THE BROWN-DOUGLAS-FILLMORE THEORY OF OPERATOR ALGEBRAS
}

BY JEROME KAMINKER AND CLAUDE SCHOCHET(l)

\begin{abstract}
The remarkable work of L. G. Brown, R. Douglas and P. Fillmore on operators with compact self-commutators once again ties together algebraic topology and operator theory. This paper gives a comprehensive treatment of certain aspects of that connection and some adjacent topics. In anticipation that both operator theorists and topologists may be interested in this work, additional background material is included to facilitate access.
\end{abstract}

0. Introduction. L. G. Brown, R. Douglas and P. Fillmore [14], [15], [16] (referred to as BDF) have forged a new link between operator theory and algebraic topology. Given a bounded operator $T \in \mathcal{L}$ with $T^{*} T-T T^{*} \in \mathcal{K}$, the compact operators, they define an element $[T]$ lying in an abelian group Ext $(X)$, where $X=\sigma(\pi T) \subset \mathbf{C}$ is the spectrum of the projection of $T$ into the Calkin algebra $\mathfrak{A}=\mathfrak{L} / \mathfrak{K}$. This class is zero if and only if $T$ is a compact perturbation of a normal operator. BDF then prove that

$$
\delta x t(X) \cong \tilde{H}^{0}(\mathrm{C}-X)
$$

the group of locally constant functions from the bounded components of $\mathbf{C}-X$ to $Z$. The explicit form of the isomorphism yields

THEOREM (BDF). Suppose $T \in \mathcal{L}$ with $T^{*} T-T T^{*}$ compact. Then $T$ is a compact perturbation of a normal operator if and only if the Fredholm index of $(T-\lambda I)$ is zero for every $\lambda$ in a bounded component of the complement of $\sigma(\pi T)$.

To carry through their proof, BDF were led to define $\delta x t(X)$ for compact metric spaces of arbitrary dimension: set $\delta \mathrm{xt}(X)$ equal to equivalence classes

Received by the editors February 19, 1975 and, in revised form, October 7, 1975.

AMS (MOS) subject classifications (1970). Primary 46L05, 47B30, 55B15, 55B20, 55H25; Secondary 47A55, 47B05, 55B40, 55J20.

Key words and phrases. $K$-theory, Steenrod homology, extensions, $C^{*}$-algebras, normal modulo the compacts, Atiyah-Hirzebruch spectral sequence, Chern character, generalized homology on compact metric spaces. 
of $C^{*}$-algebra injections $\tau: C(X) \rightarrow \mathfrak{A}$, where the relation is unitary equivalence in $\mathfrak{A}$.

BDF establish that $\delta x t(X)$ is a covariant functor from the category of compact metric spaces to abelian groups. The natural wish, then, is to relate $\operatorname{Ext}(X)$ to more common functors in topology. Let

$$
\varepsilon_{n}(X)= \begin{cases}\mathcal{E x t}(X), & n \text { odd, } \\ \mathcal{E x t}(S X), & n \text { even, }\end{cases}
$$

where $S X$ is unreduced suspension. Then $\varepsilon_{*}(X)$ satisfies the axioms for a reduced generalized homology theory (with some minor modifications). BDF also prove that on the subcategory of finite complexes, $\mathscr{E}_{*}$ is reduced homology $K$-theory $\tilde{K}_{*}$.

The purpose of this paper is to study, from the point of view of algebraic topology, the theory $\varepsilon_{*}$ on compact metric spaces. We take this to mean:

(1) an ability to compute $\varepsilon_{*}(X)$ in specific cases,

(2) developing general information about the homology theory $\varepsilon_{*}$ and its relation to $K$-theory,

(3) exploring to what extent the classical results on the Chern character and differentials in the Atiyah-Hirzebruch spectral sequence for finite complexes extend to $\mathscr{E}_{*}$ on compact metric spaces.

Our main tool is the homology theory invented by Steenrod [49]. He called it "homology based on regular cycles" but it is now called Steenrod homology. We denote it by ${ }^{s} H_{*}(X)$. There are several reasons for its relevance (aside from the after-the-fact observation that it works). First, Steenrod homology was introduced to study compact metric spaces, precisely the domain of the BDF theory. Second, the theories $\mathcal{E}_{*}$ and ${ }^{s} H_{*}$ satisfy the same axioms. This is very important. For example, Milnor [39] uses the axioms to show that the following $\lim ^{1}$ sequences are exact. Let $X$ be the inverse limit of finite complexes $X_{j}$. Then

$$
\begin{aligned}
& 0 \rightarrow \operatorname{proj} \lim ^{1} \tilde{H}_{n+1}\left(X_{j}\right) \rightarrow{ }^{s} \tilde{H}_{n}(X) \rightarrow \operatorname{proj} \lim \tilde{H}_{n}\left(X_{j}\right) \rightarrow 0, \\
& 0 \rightarrow \operatorname{proj} \lim ^{1} \varepsilon_{n+1}\left(X_{j}\right) \rightarrow \varepsilon_{n}(X) \rightarrow \operatorname{proj} \lim \varepsilon_{n}\left(X_{j}\right) \rightarrow 0
\end{aligned}
$$

are exact. The first was essentially proved by Steenrod [49].

As a last bit of evidence, the following two "Universal Coefficient Theorem" sequences are exact:

$$
\begin{aligned}
& 0 \rightarrow \operatorname{Ext}\left(\check{H}^{n+1}(X), \mathbf{Z}\right) \rightarrow{ }^{s} \tilde{H}_{n}(X) \rightarrow \operatorname{hom}\left(\check{H}^{n}(X), \mathbf{Z}\right) \rightarrow 0, \\
& 0 \rightarrow \operatorname{Ext}\left(\tilde{K}^{n+1}(X), \mathbf{Z}\right) \rightarrow \varepsilon_{n}(X) \rightarrow \operatorname{hom}\left(\tilde{K}^{n}(X), \mathbf{Z}\right) \rightarrow 0 .
\end{aligned}
$$


The first is due to Eilenberg and Mac Lane [20]. The second is due to Larry Brown [14].

Our primary contribution is the development (and use) of tools for computing $\varepsilon_{*}$ on compact metric spaces. Our concern is with compact metric spaces which in general are not $\mathrm{CW}$-complexes. In that case the spaces have no "skeleta" defined, so the Atiyah-Hirzebruch spectral sequence may not be directly applied to compute $\mathcal{E}_{*}$-homology. Nevertheless, using Steenrod homology, we prove the following theorem:

THEOREM A. Let $X$ be compact metric of dimension $d<\infty$. Then there is a spectral sequence converging to $\varepsilon_{*}(X)$, with $E^{d+1}=E^{\infty}$ and

$$
E_{p, q}^{2}={ }^{s} \tilde{H}_{p}\left(X ; \mathcal{E}_{q}\left(S^{0}\right)\right)
$$

The spectral sequence is natural in $X$.

This leads to a complete description of $\varepsilon_{*}(X)$ for $X \subset \mathbf{R}^{4}$.

THEOREM B. Let $X$ be a compact subset of $\mathbf{R}^{4}$. Then there are natural short exact sequences

$$
\begin{aligned}
& 0 \rightarrow{ }^{s} \tilde{H}_{1}(X) \rightarrow \varepsilon_{1}(X) \rightarrow{ }^{s} \tilde{H}_{3}(X) \rightarrow 0, \\
& 0 \rightarrow{ }^{s} \tilde{H}_{0}(X) \rightarrow \varepsilon_{0}(X) \rightarrow{ }^{s} \tilde{H}_{2}(X) \rightarrow 0 .
\end{aligned}
$$

The first of these sequences always splits. The splitting is natural if $X$ is a finite complex.

Our approach is axiomatic. We work almost entirely from an axiom system for a generalized Steenrod homology theory which is a modification of Milnor's system [39]. The theory $\varepsilon_{*}$ comes tailor-made for this approach.

There is an alternate approach possible. Given a representable cohomology theory on $C W$-complexes, there is an associated Steenrod homology theory on compact metric spaces. It is constructed roughly as ${ }^{s} h_{k}(X)=h^{n-k}\left(S^{n+1}-X\right)$ if $X$ is a closed subset of $S^{n+1}$. This approach has certain conceptual advantages. It is the subject of a forthcoming paper by the authors and D. S. Kahn. The axiomatic approach of the present paper leads more quickly to computations and is technically simpler, hence more palatable (we hope!) to the nontopologist.

We now outline the paper. $\$ 1$ introduces Steenrod's homology theory and the machinery of fundamental complexes, which is essential to study the algebraic topology of compact metric spaces. In \$2 Milnor's axiomatic 
description of Steenrod homology is used to define "generalized Steenrod homology"

1. Steenrod homology

2. Generalized Steenrod homology

3. The spectral sequence

4. BDF homology

5. BDF homology and $K$-theory

6. Some calculations of $\varepsilon_{*}(X)$

7. The Chern character and differentials in the spectral sequence

8. The algebraic structure of $\operatorname{Ext}(X)$. ( $\mathcal{E}_{*}$ being the example of interest). If $h_{*}$ is such a theory then Milnor's $\lim ^{1}$ sequence for a space $X=\operatorname{proj} \lim X_{j}$ reads:

$$
0 \rightarrow \mathcal{L} h_{n}(X) \rightarrow h_{n}(X) \rightarrow \check{h}_{n}(X) \rightarrow 0 \text {. }
$$

The notation is meant to imply that the groups $\mathcal{E} h_{n}(X)$ $=\operatorname{proj} \lim ^{1} h_{n+1}\left(X_{j}\right)$ and $\check{h}_{n}(X)=$ proj $\lim h_{n}\left(X_{j}\right)$ are independent of the choice of approximating spaces $X_{j}$. In $\$ 3$ the spectral sequence of Theorem A is derived for a generalized Steenrod homology theory (which the reader is free to take to be $\varepsilon_{*}$ ) and certain technical results regarding differentials are verified. $\S 4$ presents the basic results of BDF on $\varepsilon x t(X)$ and $\varepsilon_{*}(X)$. In $\S 5$ the basic connections between $\varepsilon_{*},{ }^{s} H_{*}$, and $K_{*}$ are developed. The case is made that $\mathcal{E}_{*}$ is the most natural and useful extension of homology $K$-theory to the category of compact metric spaces.

The latter sections contain applications. $\$ 6$ features an extension of the BDF low-dimensional calculations and a proof of Theorem B.

In $\$ 7$, theorems of Atiyah-Hirzebruch concerning the differentials in the spectral sequence and the Chern character are extended to $\mathcal{E}_{*}$. Here the differences between the classical situation (with finite complexes) and our present setting become most apparent.

$\S 8$ studies the algebraic structure of $\varepsilon_{*}(X)$ from the viewpoint of infinite abelian group theory. In particular, only certain abelian groups may be realized as $\mathcal{E x t}(X)$. We also relate $\mathcal{L} H_{*}$ to $\mathscr{L} \mathcal{E}_{*}$.

The authors are deeply indebted to BDF for supplying early manuscripts of their work and for a good deal of personal correspondence. This paper builds upon their original work-in particular, they were the first to see the close relations to topology.

Our k owledge of Steenrod homology is derived from Steenrod [49] and Milnor [39]. Since compact metric spaces are presently somewhat out of fashion in algebraic topology and since Milnor did not publish his paper, we have included some expository material and have reproduced some of their results. Some of these results were announced in [56]. 
Our colleagues at Indiana University have been very generous and patient in teaching us the requisite functional analysis-we are especially grateful to John Conway and Joe Stampfli for their assistance.

Analysts who wish to see the applicability of our methods should first read $\S \S 4,6,7$.

1. Steenrod homology. This section is devoted to the classical Steenrod homology theory of regular cycles and to Milnor's treatment [39] of Steenrod homology. Let eפR denote the category of compact metric spaces and continuous functions. A compact metric pair $(X, A)$ consists of a compact metric space $X$ and a closed subspace $A$.

The following definition of Steenrod homology is a variant of Steenrod's original definition (cf. Skljarenko [45]). Let $X$ be compact metric, let $K$ be an abstract countable locally finite simplicial complex with $V_{K}=$ vertices of $K$ and let $G$ be some abelian group. A regular map is a map $f: V_{K} \rightarrow X$ such that, for each $\varepsilon>0$, the images of all but finitely many simplices of $K$ have diameters less than $\varepsilon$. A regular q-chain on $X$ is a triple $\left(f, K, c_{q}\right)$, where $f: V_{K} \rightarrow X$ is a regular map and $c_{q}$ is a (possibly infinite) $q$-chain on $K$ with coefficients in $G$. The sum of two regular $q$-chains is given by the formula.

$$
\left(K, f, c_{q}\right)+\left(K^{\prime}, f^{\prime}, c_{q}^{\prime}\right)=\left(K \cup K^{\prime}, f \cup f^{\prime}, c_{q}+c_{q}^{\prime}\right) .
$$

The regular $q$-chains on $X$ with this operation and with $-\left(K, f, c_{q}\right)=(K, f$, $-c_{q}$ ) form an abelian group denoted by $C_{q}^{R}(X ; G)$.

The graded abelian group $C_{*}^{R}(X ; G)$ becomes a complex via

$$
\partial\left(K, f, c_{q}\right)=\left(K, f, \partial c_{q}\right)
$$

and reduced Steenrod homology is defined by

$$
{ }^{s} \tilde{H}_{q}(X ; G)=H_{q+1}\left(C_{*}^{R}(X ; G)\right) .
$$

If $g: X \rightarrow Y$ in $\mathbb{S T}_{\text {, then }}$ a chain map

$$
g_{\sharp}: C_{*}^{R}(X ; G) \rightarrow C_{*}^{R}(Y ; G)
$$

is defined via $g_{\sharp}\left(K, f, c_{q}\right)=\left(K, g f, c_{q}\right)$ which induces

$$
g_{*}: \tilde{H}_{*}(X ; G) \rightarrow{ }^{s} \tilde{H}_{*}(Y ; G) .
$$

The unreduced groups ${ }^{s} H_{*}(X ; G)$ are defined by ${ }^{s} H_{*}(X ; G)={ }^{s} \tilde{H}_{*}\left(X^{+} ; G\right)$, where $X^{+}$denotes $X$ with a disjoint point adjoined. Relative groups are defined in a standard way: if $(X, A)$ is a compact metric pair then let $C_{*}^{R}(X, A)=C_{*}^{R}(X) / C_{*}^{R}(A)$. Then ${ }^{s} H_{q}(X, A ; G) \cong H_{q+1}\left(C_{*}^{R}(X, A ; G)\right)$. The 
following is a known, but apparently unpublished, theorem.

TheOREM (1.2) (STEENROD). ${ }^{s} H_{*}(-; G)$ satisfies all the Eilenberg-Steenrod axioms for a homology theory.

In particular, Steenrod homology is naturally equivalent to singular and simplicial homology on the category of finite simplicial complexes. There are two additional axioms which concern us. The first is the

Relative Homeomorphism Axiom (RH): If $f:(X, A) \rightarrow(Y, B)$ is a relative homeomorphism of compact metric pairs (i.e., $f \mid X-A: X-A \rightarrow Y-B$ is a homeomorphism) then the induced map

$$
f_{*}:{ }^{s} H_{*}(X, A ; G) \rightarrow{ }^{s} H_{*}(Y, B ; G)
$$

is an isomorphism.

The statement of the second additional axiom requires some point-set topology preliminaries. Suppose $\left\{X_{j}\right\}_{j \in J}$ is a family of pointed compact metric spaces $\left(X_{j}, x_{j}\right)$. The strong wedge [40] of the family $\left\{X_{j}\right\}$, denoted $\vee_{j} X_{j}$, is the (closed!) subspace of the (compact) space $\Pi_{j} X_{j}$ of those points differing from the basepoint $\left(x_{j}\right)$ in at most one coordinate. If $J$ is a finite set this agrees with the usual wedge (defined by the weak topology) but in general the strong wedge has fewer open sets. In this paper, $\vee_{j} X_{j}$ always denotes strong wedge. Also, the set $J$ is understood to be countable unless stipulated otherwise, so that $\vee_{j} X_{j}$ is compact metric. There are canonical maps $X_{\mu} \rightarrow \vee_{j} X_{j} \rightarrow X_{\tau}$. Note that if $X$ is a compact metric space with subspaces $X_{j}$ such that $X_{i} \cap X_{j}=\left\{x_{0}\right\}$ for all $i \neq j$ and if $\operatorname{diam}\left(X_{j}\right) \rightarrow 0$ then $X \cong \vee_{j} X_{j}$. For example, the Hawaiian earring

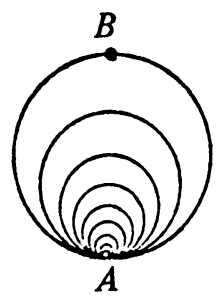

FIGURE 1

is the space $\vee_{j} S_{j}^{1}$ (the strong wedge of a countable family of circles $S^{1}$.)

The wedge axiom (W) for Steenrod homology asserts that if $\vee_{j} X_{j}$ is the strong wedge of a countable number of compact metric spaces, then the natural maps $\vee_{j} X_{j} \rightarrow X_{k}$ induce an isomorphism

$$
{ }^{s} H_{*}\left(\bigvee_{j} X_{j},\left(x_{j}\right) ; G\right) \rightarrow \prod_{j}^{s} H_{*}\left(X_{j}, x_{j} ; G\right)
$$


Milnor proves [39] that Steenrod homology is uniquely determined by Axioms $1-7, \mathrm{~W}$, and RH. Precisely,

THEOREM (1.5) (MILNOR). If $h_{*}$ is a homology theory on exl pairs satisfying the Eilenberg-Steenrod Axioms $1-17$ as well as $\mathrm{RH}$ and $\mathrm{W}$ then there exists a natural equivalence of homology theories between $h_{*}$ and ${ }^{s} H_{*}\left(-; h_{0}(\right.$ point $\left.)\right)$.

Steenrod homology is related to Cech homology as follows. Suppose $X=\operatorname{proj} \lim X_{j}$ where the $X_{j}$ are compact metric. Cech homology is continuous:

$$
\check{H}_{*}(X)=\text { proj } \lim H_{*}\left(X_{j}\right)
$$

whereas Steenrod homology is not. The following exact sequence is due to Steenrod:

$$
0 \rightarrow \operatorname{proj} \lim ^{1} H_{n+1}\left(X_{j}\right) \rightarrow{ }^{s} H_{n}(X) \rightarrow \check{H}_{n}(X) \rightarrow 0,
$$

so ${ }^{s} H_{*}$ is continuous precisely when it agrees with $\check{H}_{*}$.

REMARK (1.8). If $\left\{G_{j}, p_{j}\right\}$ is an inverse sequence of abelian groups then proj $\lim G_{j}$ and proj $\lim ^{1} G_{j}$ are defined to be the kernel and cokernel (respectively) of the homomorphism $\psi: \Pi G_{j} \rightarrow \Pi G_{j}$ defined by

$$
\psi\left(g_{0}, g_{1}, g_{2}, \ldots\right)=\left(g_{0}-p_{0} g_{1}, g_{1}-p_{1} g_{2}, \ldots\right) .
$$

The following facts are well known (cf. [4], [29], [42]).

(i) If each $G_{j}$ is finitely generated then $\operatorname{proj} \lim ^{1} G_{j}$ is either zero or an uncountable divisible group.

(ii) If $\left\{G_{j}, p_{j}\right\}$ satisfies the Mittag-Leffler condition (M-L), then $\operatorname{proj} \lim ^{1} G_{j}$ $=0$. In particular, proj $\lim ^{1} G_{j}=0$ if each $G_{j}$ is a finite-dimensional vector space over a field, or if each $G_{j}$ is a compact topological group and each $p_{j}$ is continuous.

(iii) If $0 \rightarrow\left\{A_{j}\right\} \rightarrow\left\{B_{j}\right\} \rightarrow\left\{C_{j}\right\} \rightarrow 0$ is an exact sequence of inverse sequences, then there is a 6 -term exact sequence

$$
\begin{aligned}
0 & \rightarrow \operatorname{proj} \lim A_{j} \rightarrow \operatorname{proj} \lim B_{j} \rightarrow \text { proj } \lim C_{j} \\
& \rightarrow \operatorname{proj} \lim ^{1} A_{j} \rightarrow \operatorname{proj} \lim ^{1} B_{j} \rightarrow \text { proj } \lim ^{1} C_{j} \rightarrow 0 .
\end{aligned}
$$

Steenrod developed ${ }^{s} H_{*}$ to repair the defects of Cech theory. Trial spaces for him were the solenoids which we now define. Let $G$ be an additive subgroup of the rational numbers, regarded as a discrete abelian group. The solenoid $\Sigma_{G}$ is defined to be the character group of $G$. It is a compact connected abelian 
topological group. Write $G$ as the direct limit of a directed system $\left\{\mathbf{Z}, \lambda_{i}\right\}$, where each $\lambda_{i}$ is multiplication by a power of a prime. Then

$$
\begin{aligned}
\Sigma_{G} & =\operatorname{hom}\left(G, S^{1}\right)=\text { hom }\left(\operatorname{inj} \lim \left\{\mathbf{Z}, \lambda_{j}\right\}, S^{1}\right) \\
& =\operatorname{proj} \lim \left\{\operatorname{hom}\left(\mathbf{Z}, S^{1}\right), \lambda_{j}\right\}=\operatorname{proj} \lim \left\{S^{1}, \lambda_{j}\right\},
\end{aligned}
$$

so solenoids may be regarded as inverse limits of circles. If $G=Z[1 / n]$, write $\Sigma_{n}$ for $\Sigma_{G}$. It is easy to compute ${ }^{s} H_{*}\left(\Sigma_{G}\right)$. For convenience the dyadic solenoid $\Sigma_{2}$ is illustrated.

Proposition (1.9). The homology groups of the dyadic solenoid $\Sigma_{2}$ are given by

$$
{ }^{s} \tilde{H}_{j}\left(\Sigma_{2}\right)= \begin{cases}\hat{\mathbf{Z}}_{2} / \mathbf{Z}, & j=0, \\ 0, & j>0,\end{cases}
$$

where $\hat{\mathbf{Z}}_{2}$ is the group of 2-adic integers.

Proof. If $j \geqslant 1$ then the sequence (1.7) implies ${ }^{s} H_{j}\left(\Sigma_{2}\right)=0$. The remaining case is ${ }^{s} \tilde{H}_{0}(X)$. The sequence

$$
0 \rightarrow \operatorname{proj} \lim ^{1}\{\mathbf{Z}, 2\} \rightarrow{ }^{s} \tilde{H}_{0}\left(\Sigma_{2}\right) \rightarrow \underset{\substack{\|\|}}{\tilde{H}_{0}}\left(\Sigma_{0}\right) \rightarrow 0
$$

implies that

$$
{ }^{s} \tilde{H}_{0}\left(\Sigma_{2}\right)=\operatorname{proj} \lim ^{1}\{\mathbf{Z}, 2\}
$$

reducing the rest of the proof to an exercise in algebra.

The short exact sequence of inverse sequences of abelian groups

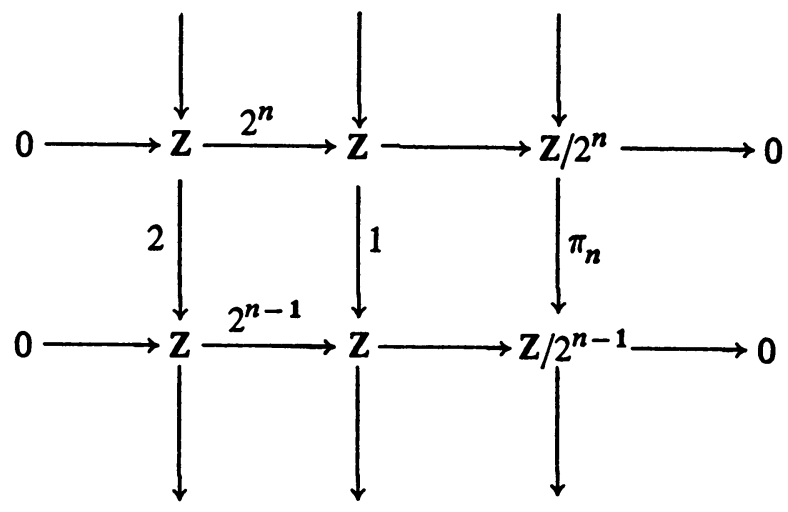


induces a 6-term lim-lim ${ }^{1}$ sequence (1.8). The relevant portion is

$$
\begin{aligned}
\operatorname{proj} \lim \{\mathbf{Z}, 2\} & \rightarrow \operatorname{proj} \lim \{\mathbf{Z}, 1\} \rightarrow \operatorname{proj} \lim \left\{\mathbf{Z} / 2^{n}, \pi_{n}\right\} \\
& \rightarrow \text { proj } \lim ^{1}\{\mathbf{Z}, 2\} \rightarrow \operatorname{proj} \lim ^{1}\{\mathbf{Z}, 1\}
\end{aligned}
$$

which is the sequence

$$
0 \rightarrow \mathbf{Z} \rightarrow \hat{\mathbf{Z}}_{2} \rightarrow \operatorname{proj} \lim ^{1}\{\mathbf{Z}, 2\} \rightarrow 0 .
$$

The group $\tilde{H}_{0}\left(\Sigma_{2}\right)=\hat{\mathbf{Z}}_{2} / \mathbf{Z}$ is uncountable and divisible. In general, if $G$ is a connected compact metric abelian group, then ${ }^{s} \tilde{H}_{0}(G)$ is in 1-1 correspondence with the set of path components of $G$.

REMARK (1.10). Singular theory is also ill-behaved on eפT. Milnor and Barratt [40] show that the singular homology of $V_{j} S_{j}^{2}$ (strong wedge) is nonzero in infinitely many dimensions (despite the fact that $V_{j} S_{j}^{2}$ is a compact subset of $\left.S^{3}\right)$. But ${ }^{s} H_{*}\left(\bigvee_{j} S_{j}^{2}\right)=\prod_{j} H_{*}\left(S_{j}^{2}\right)$ by $(\mathrm{W})$, so ${ }^{s} H_{k}\left(\bigvee_{j} S_{j}^{2}\right)$ $=0$ for $k \geqslant 3$.

The remaining part of this section is devoted to fundamental complexes. These provide a key technical tool in the study of eפtr. We follow Milnor's presentation.

Suppose $X$ is compact metric and $\left\{\mathscr{U}_{i}\right\}_{i \geqslant 0}$ is a sequence of finite open covers of $X$ with $\mathcal{Q}_{0}=\{X\}, \mathcal{U}_{i}$ refining $\mathcal{Q}_{i-1}$, and mesh $\left(\mathcal{U}_{i}\right) \rightarrow 0$. Associated to this data is a convergent nerve system (CNS) $\underline{X}=\left\{X_{j}, p_{j}\right\}$ which is constructed as follows. Let $X_{i}$ be the geometric realization of $\mathscr{Q}_{i}$. Then $X_{i}$ is a finite simplicial complex. The maps $p_{i}: X_{i+1} \rightarrow X_{i}$ are defined as follows. If $v \in X_{i+1}$ is a vertex of $X_{i+1}$ corresponding to an open set $V \in \mathcal{Q}_{i+1}$, let $p_{i}(v)$ be the barycenter of that simplex of $X_{i}$ which is spanned by the vertices corresponding to those open sets of $U_{i}$ containing $V$. Then $p_{i}$ is defined on the vertices of $X_{i+1}$ and extends linearly to a continuous function $X_{i+1} \rightarrow X_{i}$. Note that $p_{i}$ sends vertices to barycenters, so that $p_{i}: X_{i+1} \rightarrow X_{i}^{\prime}$ is simplicial (where $X_{i}^{\prime}$ is the barycentric subdivision of $X_{i}$ ).

The CNS $X$ satisfies two important properties. First, $X=\operatorname{proj} \lim X_{j}$. Second, given $\varepsilon>0$ and $i \geqslant 0$, there exists $j \geqslant i$ such that $p_{i} \circ \cdots \circ p_{j-1}: X_{j}$ $\rightarrow X_{i}$ maps each simplex into a set of diameter less than $\varepsilon$. This "convergence" condition makes possible the naturality of the following constructions, which is essential.

Given a compact metric space $X$ and CNS $\underline{X}=\left\{X_{j}, p_{j}\right\}$, there is associated a countable locally finite, contractible $C W$-complex $F=F(\underline{X})$, constructed as follows. Let $M_{i}$ be the mapping cylinder of $p_{i}$. It admits the structure of a finite complex with $X_{i}$ and $X_{i+1}$ as subcomplexes. Identify $M_{i}$ and $M_{i+1}$ along their common part $X_{i+1}$ (this requires barycentric subdividing $X_{i+1}$ in $M_{i}$ and 
extending this to a subdivision of $M_{i}$ without changing $\left.X_{i}\right)$. Let $F_{0}=$ (point $\}$ and for $i \geqslant 1$, let

$$
F_{i}=M_{0} \cup M_{1} \cup \cdots \cup M_{i-1} / \sim
$$

and

$$
F=F(\underline{X})=\bigcup_{i>0} F_{i} .
$$

Then $F(X)$ is a countable locally finite CW-complex, the fundamental complex of the CNS $X$.

Let $T(X)=\operatorname{proj} \lim \left\{F_{i}, \rho_{i}\right\}$, where $\rho_{i}: F_{i+1} \rightarrow F_{i}$ is the obvious retraction.

Proposition (1.13) (MILNor). Let $\underline{X}$ be a CNS for $X$. Then

(1) $T(\underline{X})$ is a contractible compact metric space.

(2) There is an embedding $F(\underline{X}) \rightarrow T(\underline{X})$ with $F(\underline{X})$ an open, dense subset of $T(X) . T(X)-F(X) \cong X$.

(3) $T(\underline{X}) \backslash F(\underline{X}) \cong X$.

Now a bit of notation. If $X$ is a locally compact Hausdorff space, let $\dot{X}$ denote its one-point compactification. A proper map $f: X \rightarrow Y$ induces $\dot{f}: \dot{X} \rightarrow \dot{Y}$. Note that if $X$ is a countable, locally finite cw-complex, then $\dot{X}$ is compact metric.

Proposition (1.14). Let $\underline{X}$ be a CNS for $X$. Let $F^{j} \subset F(\underline{X})$ be the j-skeleton of the fundamental complex. Then

(1) $\dot{F}^{p} / \dot{F}^{p-1} \cong \bigvee_{j \in J_{p}} S_{j}^{p}, J_{p}$ is the set of p-cells of $F(\underline{X})$.

(2) $T(X) / X \cong F(\underline{X})$.

REMARK (1.15). The exact sequence of the pair $(T(X), X)$ together with (1.13) (1) and (1.14) (2) allows one to identify ${ }^{s} \tilde{H}_{j}(F(X)) \cong{ }^{s} \tilde{H}_{j-1}(X)$. This carries over to generalized Steenrod homology theories.

The following proposition uses the convergence condition for the first time.

Proposition (1.16) (MILNOR). Let $X$ and $Y$ be compact metric spaces with CNS $X, Y$ respectively, and let $f: X \rightarrow Y$. Then there is a map of triples

$$
\bar{f}:(T(\underline{X}), F(\underline{X}), X) \rightarrow(T(Y), F(\underline{Y}), Y)
$$

with $\bar{f} \mid X=f$, and the map $\bar{f} \mid F(\underline{X})$ is a proper cellular map, inducing

$$
F(f): \dot{F}(\underline{X}) \rightarrow \dot{F}(\underline{Y}) .
$$

RemarK (1.17). Milnor showed [39] that Čech cohomology may be obtained as the homology of a certain cochain complex and that the homology of the dual chain complex was Steenrod homology. This immediately yields the Universal Coefficient Theorem (0.5) 


$$
0 \rightarrow \operatorname{Ext}\left(\check{H}^{n+1}(X), \mathbf{Z}\right) \rightarrow{ }^{s} H_{n}(X) \rightarrow \operatorname{hom}\left(\check{H}^{n}(X), \mathbf{Z}\right) \rightarrow 0
$$

due originally to Eilenberg and Mac Lane [20].

RemarK (1.18). We indicate here how classical Steenrod homology is related to other known theories. On the category of compact metric spaces, BorelMoore homology [11] is equivalent to Steenrod homology. The best-known theories are singular and Čech. There are natural transformations

$$
H_{*}^{\text {sing }} \stackrel{\oplus}{\rightarrow} H_{*} \stackrel{\Psi}{\longrightarrow} \check{H}_{*}
$$

where $\phi$ is obtained by an acyclic models argument and $\psi$ is the map which occurs in the $\lim ^{1}$-sequence (1.7). Since $\psi$ is onto and (1.7) describes $\operatorname{ker}(\psi)$ it is quite useful to us. The map $\phi$ is less useful, as the Barratt-Milnor example (1.10) illustrates.

2. Generalized Steenrod homology. This section is devoted to the axiomatic development of the properties of a generalized Steenrod homology theory $h_{*}$. (The word "generalized" indicates that the dimension axiom has been omitted.) The basic examples are the BDF theory $\varepsilon_{*}$ and Steenrod homology ${ }^{s} \tilde{H}_{*} \cdot \lim ^{1}$ Fundamental complexes are used to prove 's Theorem (2.7). The lim ${ }^{1}$ subgroup $\mathcal{L} h_{n}(X) \subset h_{n}(X)$ is then studied in some detail. In many cases it vanishes; sometimes it does not. Either way it represents how $\mathcal{E}_{*}$ differs from the Čech extension $\breve{K}_{*}$ of homology $K$-theory to compact metric spaces and is thus of considerable interest. As a spin-off of our study we show that the natural map ${ }^{s} H_{*}(X ; G) \rightarrow \check{H}_{*}(X ; G)$ is an isomorphism when $G$ is algebraically compact, and hence $\breve{H}_{*}(-; G)$ is a homology theory. This generalizes the well-known Eilenberg-Steenrod results [21].

Definition (2.1). A generalized Steenrod homology theory consists of a sequence $h_{*}=\left\{h_{n} \mid n \in \mathbf{Z}\right\}$ of covariant, homotopy invariant functors from the cateogry exR of compact metric spaces to abelian groups satisfying the following axioms:

(E) If $(X, A)$ is a compact metric pair, then the natural sequence

$$
h_{n}(A) \rightarrow h_{n}(X) \rightarrow h_{n}(X / A)
$$

is exact for all $n$.

(S) There is a sequence of natural equivalences

$$
\sigma_{n}: h_{n} \rightarrow h_{n+1} \circ S
$$

called suspension, where $S$ is unreduced suspension.

(W) If $X=\bigvee_{j=1}^{\infty} X_{j}$ is the strong wedge of a sequence of pointed compact metric spaces then the natural projections $X \rightarrow X_{j}$ induce an isomorphism 


$$
h_{*}(X) \rightarrow \Pi_{j} h_{*}\left(X_{j}\right) .
$$

We list three examples of such theories. First, note that Steenrod homology theory ${ }^{s} \tilde{H}_{*}(X ; G)$ satisfies (E), (S) and (W), and is thus a generalized Steenrod homology theory.

The second example is (reduced) Čech homology theory [21], with appropriate coefficients, denoted by $\check{H}_{n}(X ; G)$. (Note that the tilde is left off $H_{n}$ for simplicity.) For arbitrary groups $G$, this theory satisfies all the axioms except exactness. Eilenberg and Steenrod show that exactness is satisfied when $G$ is compact or a vector space over a field, but not for $G=\mathbf{Z}$. Later in this section we show that $\breve{H}_{*}(X ; G)$ is exact and hence a generalized Steenrod homology theory when $G$ is algebraically compact. In that case it is naturally equivalent to ${ }^{s} \tilde{H}_{*}(X ; G)$.

The third example is Brown-Douglas-Fillmore homology $\varepsilon_{*}(X)$, defined by

$$
\varepsilon_{n}(X)= \begin{cases}\varepsilon \operatorname{xt}(X), & n \text { odd, } \\ \varepsilon x t(S X), & n \text { even } .\end{cases}
$$

This is discussed in detail in $\S 4$; it is the focus of this paper.

There are a number of very elementary consequences of the axioms. These vary in occasionally consequential ways from the usual properties of a generalized homology theory, so we list them.

Proposition (2.2). $h_{n}$ (point) $=0$ for all $n$.

This is immediate from exactness applied to the pair (point, point). Thus the theories considered here are "reduced" but yet not defined on a pointed category. The coefficients of a generalized Steenrod homology theory are $h_{*}\left(S^{0}\right)$. Note that

$$
{ }^{s} \tilde{H}_{j}\left(S^{0} ; G\right)= \begin{cases}G, & j=0, \\ 0, & j \neq 0,\end{cases}
$$

and

$$
\varepsilon_{j}\left(S^{0}\right)= \begin{cases}\mathbf{Z}, & j \text { even } \\ 0, & j \text { odd }\end{cases}
$$

Proposition (2.3). Let $(X, A)$ be a compact metric pair with $A$ contractible. Then the natural map $h_{*}(X) \rightarrow h_{*}(X / A)$ is an isomorphism.

Both (2.3) and the following Proposition (2.4) are proved in [15] for $\mathcal{E}_{*}$; their proofs hold in general. A Barratt-Puppe sequence type of argument and (2.3) imply (2.4), but note that no cofibration requirement is stipulated for (2.4). 
Proposition (2.4). Let $(X, A)$ be a compact metric pair with $A \neq \varnothing$. Then there is a natural long exact sequence

$$
\cdots \rightarrow h_{n}(A) \rightarrow h_{n}(X) \rightarrow h_{n}(X / A) \rightarrow h_{n-1}(A) \rightarrow \cdots
$$

If $S X$ denotes unreduced suspension and $\underline{S} X$ denotes reduced suspension (with any point of $X$ taken as basepoint) then the quotient map $S X \rightarrow \underline{S} X$ induces an isomorphism

$$
h_{*}(S X) \rightarrow h_{*}(\underline{S} X)
$$

by (2.3). This is true despite the fact that varying the choice of basepoint may alter the homotopy type of $\underline{S} X$. For example, if $A$ and $B$ are the two points indicated on the Hawaiian earring $W$ in Figure 1 , then $\underline{S}_{A} W$ is not homotopy equivalent to $\underline{S}_{B} W$. The difference is detected by singular homology, for ${ }^{\text {sing }} H_{3}\left(\underline{S}_{A} W\right) \neq 0$ while ${ }^{\text {sing }} H_{3}\left(S_{B} W\right)=0$ (see (1.10)), but not by Steenrod homology theory.

Proposition (2.5). Let $X$ be compact metric. Let $X$ be a CNS for $X$ with fundamental complex $F$ and "compactification" $T$. Then $h_{n}(\dot{F})=h_{n-1}(X)$.

Proof. Apply (2.4) to the pair $(T, X)$ and use the fact that $T$ is contractible and $T / X \cong \dot{F}$.

This proposition is the key to the construction of the spectral sequence. The space $X$ has no natural filtration but the space $F$ does.

Proposition (2.6). Let $(X ; A, B)$ be a triad of compact metric spaces with $X=A \cup B$ and $A \cap B \neq \varnothing$. Then there is a natural Mayer-Vietoris sequence

$$
\begin{aligned}
\cdots & \rightarrow h_{n}(A \cap B) \stackrel{\left(i_{10},-i_{2_{0}}\right)}{\longrightarrow} h_{n}(A) \oplus h_{n}(B) \stackrel{\left(j_{l_{0}}+j_{2_{0}}\right)}{\longrightarrow} h_{n}(X) \rightarrow h_{n-1}(A \cap B) \\
& \rightarrow \cdots .
\end{aligned}
$$

The proof is standard; see [21]. We now obtain a version of Milnor's limsequence.

TheOREM (2.7) (MILNOR [39]) . Let $X$ be compact metric and let $X$ be a CNS for $X$. Let $h_{*}$ be a generalized Steenrod homology theory. Then there is an exact sequence

$$
0 \rightarrow \operatorname{proj} \lim ^{1} h_{n+1}\left(X_{j}\right) \rightarrow h_{n}(X) \rightarrow \operatorname{proj} \lim h_{n}\left(X_{j}\right) \rightarrow 0
$$

Proof. Let $F$ be a fundamental complex for $\underline{X}$. Set 


$$
\begin{aligned}
& F_{o}=M_{1} \cup M_{3} \cup M_{5} \cup \cdots, \\
& F_{e}=M_{0} \cup M_{2} \cup M_{4} \cup \cdots .
\end{aligned}
$$

Then

$$
\begin{aligned}
\dot{F} & =\dot{F}_{o} \cup \dot{F}_{e}, \\
\dot{F}_{o} \cap \dot{F}_{e} & =\bigvee_{j \geqslant 1}^{\bigvee} X_{j}^{+} \quad\left(Y^{+}=Y \text { with disjoint basepoint, }\right) \\
\dot{F}_{o} & =\bigvee_{j \geqslant 0} M_{2 j+1}^{+}, \quad \dot{F}_{e}=\bigvee_{j \geqslant 0}^{\bigvee} M_{2 j}^{+} .
\end{aligned}
$$

Apply Proposition (2.6) and (W) to $\left(\dot{F}, \dot{F}_{o}, \dot{F}_{e}\right)$ to obtain the commutative diagram

$$
\prod_{j>1} \prod_{n+1}\left(X_{j}^{+}\right) \stackrel{\psi_{n+1}}{\longrightarrow} \prod_{j>0} h_{n+1}\left(\dot{F}_{o}^{+} \cap \dot{F}_{e}\right) \rightarrow h_{n+1}\left(\dot{F}_{0}\right) \oplus h_{n+1}\left(\dot{F}_{e}\right) \rightarrow h_{n+1}(\dot{F}) \rightarrow \cdots
$$

Some careful identifications and the serpent lemma imply that

$$
\operatorname{ker} \psi_{n+1} \cong \operatorname{proj} \lim h_{n+1}\left(X_{j}\right), \quad \operatorname{cok} \psi_{n+1} \cong \operatorname{proj} \lim ^{1} h_{n+1}\left(X_{j}\right)
$$

Then the sequence

$$
\begin{aligned}
& 0 \rightarrow \operatorname{proj} \lim ^{1} h_{n+1}\left(X_{j}\right) \rightarrow h_{n+1}(\dot{F}) \rightarrow \operatorname{proj} \lim h_{n}\left(X_{j}\right) \rightarrow 0 \\
& \text { IR } \\
& h_{n}(X)
\end{aligned}
$$

yields the theorem.

REMARK (2.8). Milnor shows that if $X=\operatorname{proj} \lim X_{j}$ for any inverse sequence of compact metric spaces $X_{j}$ then the above theorem still holds.

Definition (2.9). A natural transformation of generalized Steenrod homology theories is a sequence of natural transformations $h_{n} \rightarrow h_{n}^{\prime}$ which commute with suspension.

Proposition (2.10). Let $\eta: h_{*} \rightarrow h_{*}^{\prime}$ be a natural transformation of Steenrod homology theories and let $\underline{X}$ be a $C N S$ for the compact metric space $X$. Then there is a commutative diagram 
$0 \longrightarrow \operatorname{proj} \lim ^{1} h_{n+1}\left(X_{j}\right) \longrightarrow h_{n}(X) \longrightarrow \operatorname{proj} \lim h_{n}\left(X_{j}\right) \rightarrow 0$

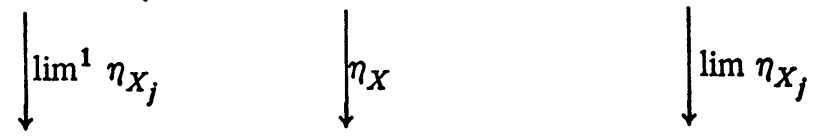

$0 \longrightarrow \operatorname{proj} \lim ^{1} h_{n+1}^{\prime}\left(X_{j}\right) \longrightarrow h_{n}^{\prime}(X) \longrightarrow \operatorname{proj} \lim h_{n}^{\prime}\left(X_{j}\right) \rightarrow 0$

The proof is immediate.

Proposition (2.11). The $\lim ^{1}$-sequence (2.7) is natural with respect to maps.

Proof. Let $f: X \rightarrow X^{\prime}$ be a map of compact metric spaces and let $X$ and $X^{\prime}$ be CNS for $X, X^{\prime}$ respectively. There is a cofinal CNS $X$ of $X$ such that the map

$$
\bar{f}:(T(\underline{X}), F(\underline{X}), X) \rightarrow\left(T\left(\underline{X}^{\prime}\right), F\left(\underline{X}^{\prime}\right), X^{\prime}\right)
$$

obtained via (1.16) preserves mapping cylinders. Then $F(f)$ induces a map of triads

$$
F(f):\left(F ; F_{o}, F_{e}\right) \rightarrow\left(F^{\prime} ; F_{o}^{\prime}, F_{e}^{\prime}\right)
$$

and hence a commutative diagram

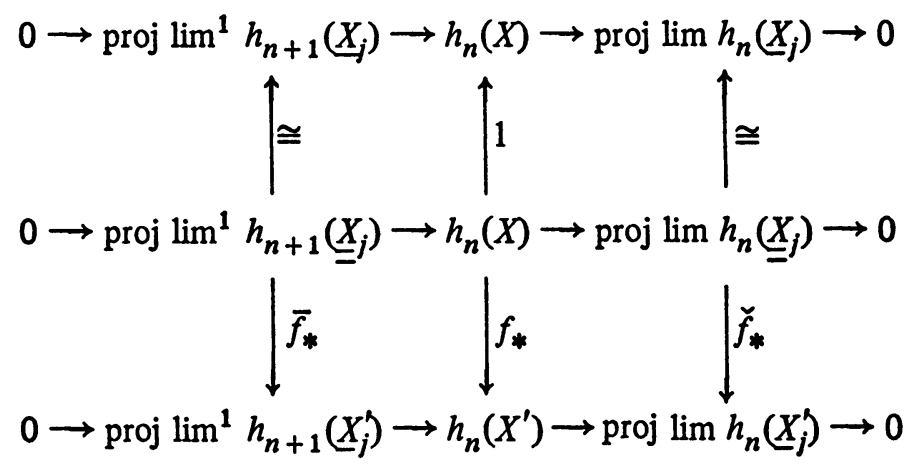

Composing the vertical maps yields the desired diagram.

Corollary (2.12). The group $\lim ^{1} h_{n+1}\left(\underline{X}_{j}\right)$ is independent of CNS. Denote it by $\mathcal{L} h_{n}(X)$. Then $\mathrm{L} h_{n}(-)$ is homotopy invariant on $\mathrm{eNT}$

Proof. Apply (2.11).

Proposinion (2.13). Suppose $h_{*}$ is a generalized Steenrod homology theory with $h_{*}\left(S^{0}\right)$ of finite type. Then $\mathcal{L} h_{n}(X)$ is divisible and the sequence 


$$
0 \rightarrow \mathfrak{L} h_{n}(X) \rightarrow h_{n}(X) \rightarrow \check{h}_{n}(X) \rightarrow 0
$$

splits unnaturally.

Proof. A Mayer-Vietrois argument shows that $h_{*}\left(X_{j}\right)$ is of finite type for

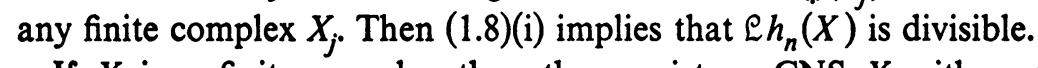

If $X$ is a finite complex then there exists a CNS $\underline{X}$ with $p_{j_{*}}: h_{*}\left(X_{j+1}\right)$ $\rightarrow h_{*}\left(X_{j}\right)$ an isomorphism for all $j \geqslant 1$. Thus $\left\{h_{*}\left(X_{j}\right), p_{j_{*}}\right\}$ satisfies M-L (see (1.8)(ii)) and so $\mathcal{L} h_{*}(X)=0$.

REMARK (2.14). The theory of shape, due to Borsuk, provides a classification of compact metric spaces which is coarser than homotopy equivalence [12]. In this theory there is the notion of movable compacta. These are a class of spaces more general than ANR's but less pathological than arbitrary compact metric spaces. If $X$ is a movable metric compactum, then $\left\{h_{*}\left(X_{j}\right), p_{j_{*}}\right\}$ satisfies M-L for any CNS $X$ [36]. Thus $X$ movable implies that $h_{n}(X)=h_{n}(X)$.

Mardešić shows [34] that if $X$ is $n$-dimensional and $(n-1)$ homologically locally connected, then $X$ is movable. We mention this since homological local connectivity has been used in a related context by Ekman [22].

Nonzero elements of $\mathfrak{L} h_{n}(X)$ are the homology analogue of "phantom" cohomology classes-that is, classes $x \in h^{*}(X)$, where $X$ is an infinite CWcomplex, which are nonzero but which vanish upon restricting to finite skeleta of $X$. The corresponding fact for "phantom" homology classes is given by the following proposition.

Proposirion (2.15). Let $h_{*}$ be a Steenrod homology theory, and let $X$ be compact metric embedded in the Hilbert cube $I^{\omega}$. Then a class $x \in h_{n}(X)$ is in the subgroup $\mathfrak{L} h_{n}(X)$ if and only if for every neighborhood $U$ of $X$ in $I^{\omega}$, the induced $\operatorname{map}_{n}(X) \rightarrow h_{n}(\bar{U})$ sends $x$ to zero.

Proof. Let $\left\{X_{j}\right\}$ be a sequence of compact ANR's with $X_{j} \subseteq I^{\omega}, X$ $\subset$ int $\left(X_{j}\right), X=\cap_{j} X_{j}$ and $X_{j} \subseteq X_{j+1} \subseteq \cdots$. The sequence $\left\{X_{j}\right\}$ forms an ANR system $X$ in the sense of Mardešić-Segal and so, also, does any CNS $X=\left\{X_{j}^{\prime}\right\}$ for $X$. By [37, Theorem 12] there exists a homotopy equivalence of ANR systems $f: \underline{X} \rightarrow X$ making the natural diagram

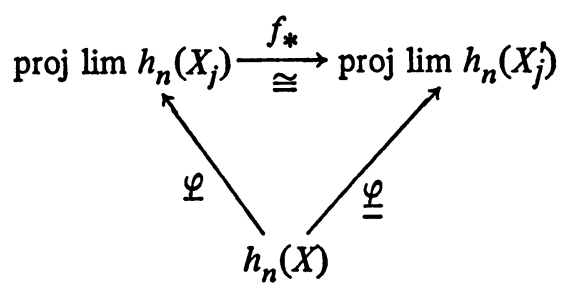


commute. Thus $\mathcal{L} h_{n}(X) \cong \operatorname{ker} \varphi \cong \operatorname{ker} \underline{\varphi}$. Let $\bar{V}$ be a closed neighborhood of $X$ in $I^{\omega}$. Then there exists an $n$ with $X \subseteq X_{n} \subseteq V$ and the result follows.

The above proof shows that $\check{h}_{n}(X)=$ proj $\lim h_{n}\left(X_{j}\right)$ where $\left\{X_{j}\right\}$ is any ANR system, and similarly for $\mathcal{L} h_{n}(X)$.

REMARK (2.16). The subgroup $\mathcal{L} h_{n}(X)$ may also be characterized as those elements $x \in h_{n}(X)$ such that $g_{*}(x)=0$ for all maps $g: X \rightarrow Y, Y$ a finite complex.

COROLlary (2.17). Let $h_{*}$ and $h_{*}^{\prime}$ be generalized Steenrod homology theories with coefficients of finite type, and suppose $h_{*}$ is naturally equivalent to $h_{*}^{\prime}$ on the category of finite complexes. Then, for any compact metric space $X, h_{*}(X)$ $\cong h_{*}^{\prime}(X)$ (unnaturally .)

Proof. Apply (2.13).

REMARK (2.18). Corollary (2.17) is a very weak uniqueness theorem. In the case of ordinary Steenrod homology, Milnor [39] produces an equivalence of homology theories $h_{*} \rightarrow h_{*}^{\prime}$. His proof relies upon the fact that ${ }^{s} H_{*}(X)$ may be described as $H\left(C_{*}^{\infty}(F(\underline{X}))\right)$. If one were given a natural transformation $\Re: h_{*} \rightarrow h_{*}^{\prime}$ which was an equivalence on finite complexes then a spectral sequence argument would allow one to conclude that $\pi$ was an equivalence on finite-dimensional compact metric spaces. However, the question at hand is whether the axioms uniquely characterize the Steenrod extension of a generalized homology theory. This is unknown to us.

Definition (2.19) [23, pp. 159-168]. An abelian group is algebraically compact if it is algebraically a direct summand of a group that admits a compact topology.

For example, divisible groups and compact groups are algebraically compact.

TheOREM (2.20). Suppose $G$ is an algebraically compact group. Then so is $H_{n}\left(X_{j} ; G\right)$ for a finite complex $X$ and ${ }^{s} H_{*}(Y ; G)=0$ for any compact metric space $Y$.

Proof. Write $G \oplus H=C$, where $C$ is compact and $X=\operatorname{proj} \lim X_{j}$ where $X_{j}$ are finite complexes. Then

$$
{ }^{s} H_{n}(Y ; C)={ }^{s} H_{n}(Y ; G) \oplus{ }^{s} H_{n}(Y ; H)
$$

for any space $Y$ in $\operatorname{Cr}$. The group $H_{n}\left(X_{j} ; C\right)$ is compact; hence $H_{n}\left(X_{j} ; G\right)$ is algebraically compact. Further,

$$
\mathcal{L} \tilde{H}_{*}(X ; C)=0
$$

since the sequence 


$$
\rightarrow H_{n}\left(X_{j} ; C\right) \rightarrow H_{n}\left(X_{j-1} ; C\right) \rightarrow
$$

is a sequence of compact groups and continuous homomorphisms (1.8)(ii). But (2.21) implies that

$$
\mathfrak{L}^{s} H_{*}(X ; C)=\mathfrak{L}^{s} H_{*}(X ; G) \oplus \mathfrak{L}^{s} H_{*}(X ; H)
$$

and then (2.22) implies that $\mathfrak{L}^{s} H_{*}(X ; G)=0$.

COROLlaRY (2.23). Cech homology with coefficients in an algebraically compact group $G$ satisfies the exactness axiom and hence is a homology theory on $\mathrm{CNT}_{\text {. }}$

Proof. ${ }^{s} H_{*}(X ; G)=\check{H}_{*}(X ; G)$.

We close this section with a comment upon notation. Let $h_{*}$ be a generalized homology theory on finite complexes. Then it corresponds (via Spanier-Whitehead duality) to a cohomology theory $h^{*}$ on finite complexes. E. Brown's representability theorem guarantees (with mild hypotheses) that

$$
h^{n}(X)=\left[X, E_{n}\right]
$$

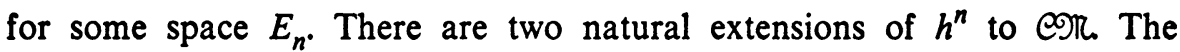
"representable" theory uses (2.24) as a definition. The "Čech" extension is obtained by taking a cofinal sequence of nerves of finite covers, realizing them by finite complexes $X_{j}$, and setting $\check{h}^{n}(X)=\operatorname{proj} \lim h^{n}\left(X_{j}\right)$. In fact this is equivalent to (2.24) [32], so there is no ambiguity.

Note that for cohomology $K$-theory, $K^{0}(X)$ may also be defined directly for compact spaces [6]. This functor is continuous [43]. Hence it is equivalent to the Čech extension $K^{0}$ of $K$-theory from finite complexes.

3. The spectral sequence. In this section a spectral sequence converging to $h_{*}(X)$ is constructed, where $h_{*}$ is a generalized Steenrod homology theory and $X$ is compact metric of finite dimension. It will be shown that

$$
E_{p, q}^{2}={ }^{s} \tilde{H}_{p}\left(X ; h_{q}\left(S^{0}\right)\right) .
$$

Various naturality considerations are examined. In subsequent sections the spectral sequence will be applied to the case $h_{*}=\varepsilon_{*}$, the BDF homology theory. The construction parallels that of the Atiyah-Hirzebruch spectral sequence but with major differences since compact metric spaces do not come equipped with an obvious filtration.

Let $X$ be compact metric and let $X$ be a CNS for $X$, with corresponding fundamental complex $F$. The space $F$ is a CW-complex so it is filtered by its skeleta $F^{i}$, and the one-point compactification $F$ is filtered by closed subspaces $\dot{F}^{i}$. Following Massey [38], form an exact couple, with 


$$
E_{p, q}^{1}=h_{p+q+1}\left(\dot{F}^{p+1} / \dot{F}^{p}\right), \quad D_{p, q}^{1}=h_{p+q+1}\left(\dot{F}^{p+1}\right),
$$

and the maps

$$
\begin{aligned}
& i_{p, q}: D_{p, q}^{1} \rightarrow D_{p+1, q-1}^{1}, \\
& j_{p, q}: D_{p, q}^{1} \rightarrow E_{p, q}^{1}, \\
& k_{p, q}: E_{p, q}^{1} \rightarrow D_{p-1, q}^{1},
\end{aligned}
$$

which are obtained from the exact sequences of the appropriate pairs. Let $\left(E_{p, q}^{r}, d_{p, q}^{r}\right)$ be the associated spectral sequence. (When we wish to indicate the (seeming) dependence upon $X$ we use $E_{p, q}^{r}(\underline{X})$.) Then each $E^{r}$ is a bigraded group, $d_{r}: E_{p, q}^{r} \rightarrow E_{p-r, q+r-1}^{r}$, and $H\left(E^{r}\right)=E^{r+1}$.

There are now several tasks. One must relate the limit term of the spectral sequence to $h_{*}(X)$, the $E^{2}$ term to Steenrod homology, remove the dependence upon the choice of $X$, and check various naturality claims.

Recall from (2.5) that $h_{p+q+1}(\dot{F})$ is naturally isomorphic to $h_{p+q}(X)$. Define $\mathscr{F}^{p, q}$ to be the image of the composite

$$
h_{p+q+1}\left(\dot{F}^{p+1}\right) \rightarrow h_{p+q+1}(\dot{F}) \stackrel{\cong}{\rightrightarrows} h_{p+q}(X) .
$$

Then

$$
\begin{aligned}
0 & =\mathscr{F}^{-1, p+q+1} \subset \mathscr{F}^{0, p+q} \subset \mathscr{F}^{1, p+q-1} \subset \cdots \\
& \subset \mathscr{F}^{k, p+q-k} \subset \cdots \subset h_{p+q}(X)
\end{aligned}
$$

provides a filtration of $h_{p+q}(X)$.

Proposition (3.3). The filtration $\mathscr{F}^{p, q}$ in (3.2) is independent of the choice of CNS. If $X$ has finite dimension $d$ then

$$
E_{p, q}^{d+1} \cong E_{p, q}^{\infty} \cong \mathscr{F}^{p, q} / \mathscr{F}^{p-1, q+1}
$$

Proof. Suppose that $\underline{X}, \underline{X}$ are two CNS's for $X$. By (1.16), the identity map on $X$ induces a map of triples

$$
\text { g: }(T, F, X) \rightarrow(\underline{T}, \underline{F}, X)
$$

with $g \mid X=1_{X}$ and $g \mid F$ a proper cellular map. Then $g_{*}: h_{p+q+1}(\dot{F})$ $\rightarrow h_{p+q+1}(\dot{F})$ is filtration-preserving and the diagram 


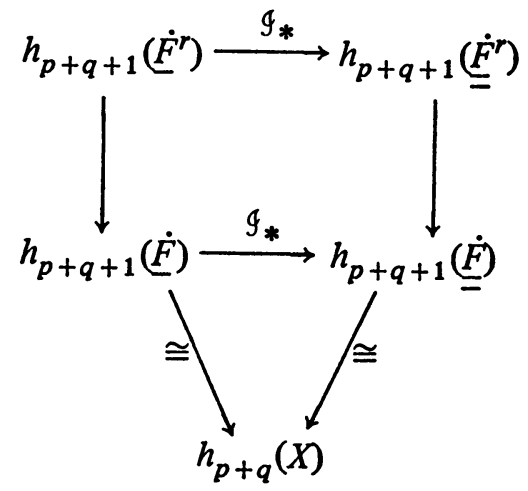

commutes, showing the independence of $\mathscr{F}^{p, q}$.

If $\operatorname{dim}(X)=d$ then proj $\lim _{r} h_{p+q+1}\left(\dot{F}^{r}\right)=h_{p+q+1}(\dot{F})$. In fact, $\dot{F}^{d+1}=\dot{F}$, so $\mathscr{F}^{d, p+q-d}=h_{p+q}(X)$ and the proposition follows.

Proposition (3.4). Suppose $X$ and $Y$ are compact metric with $C N S X, Y$ respectively. Let $f: X \rightarrow Y$. Then there is a morphism of spectral sequences $f_{r}: E^{r}(\underline{X}) \rightarrow E^{r}(\underline{Y})$ which is covered on the $E^{\infty}$ level by $f_{*}: h_{*}(X) \rightarrow h_{*}(Y)$.

Proof. By (1.16), $f$ induces a map of triples $f:(T, F, X) \rightarrow\left(T^{\prime}, F^{\prime}, Y\right)$ with $f \mid X=f$ and $f \mid F$ a proper cellular map. Then $\underline{f}$ induces a morphism of exact couples and hence a morphism of spectral sequences covering $f_{*}$.

REMARK (3.5). If $\eta: h_{*} \rightarrow h_{*}^{\prime}$ is a natural transformation of generalized Steenrod homology theories, then the map $h_{*}(X) \rightarrow h_{*}^{\prime}(X)$ is covered by a morphism of spectral sequences.

Next, the $E^{2}$ term is identified. The result will imply that the spectral sequence $E_{p, q}^{r}(X)$ is independent of the choice of CNS from $E^{2}$ onward (justifying the notation $\left.E_{p, q}^{r}(X)\right)$ and that the morphisms (3.4) are canonically determined.

Proposition (3.6). $E_{p, q}^{2}(\underline{X})$ is naturally isomorphic to ${ }^{s} \tilde{H}_{p}\left(X ; h_{q}\left(S^{0}\right)\right)$ via $\theta_{X}$ (to be defined).

Proof. First observe that the chain complex $\left\{E_{p, q}^{1}, d_{p, q}^{1}\right\}$ is precisely the infinite cellular chain complex of the countable locally finite CW-complex $F$, with coefficients in $h_{q}\left(S^{0}\right)$. That is, if $J_{n}$ is an index set for the $n$-cells of $F$, then

$$
\begin{aligned}
& E_{p, q}^{1}=h_{p+q+1}\left(\dot{F}^{p+1} / \dot{F}^{p}\right)=h_{p+q+1}\left(\bigvee_{p+1} S^{p+1}\right) \\
& \cong \prod_{J_{p+1}} h_{q}\left(S^{0}\right)=C_{p+1}^{\infty}\left(\dot{F} ; h_{q}\left(S^{0}\right)\right)
\end{aligned}
$$

and the boundary map $d_{p, q}^{1}$ is defined so that 


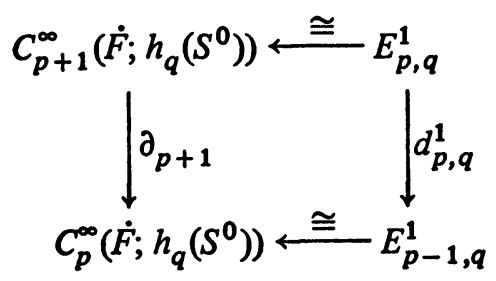

commutes. Thus there is an isomorphism

$$
E_{p, q}^{2} \cong H_{p+1}\left(C_{*}^{\infty}\left(F ; h_{q}\left(S^{0}\right)\right)\right)
$$

which is natural for proper cellular maps. Milnor [39] shows that

$$
H_{p+1}\left(C_{*}^{\infty}\left(F ; h_{q}\left(S^{0}\right)\right)\right) \cong{ }^{s} \tilde{H}_{p+1}\left(F ; h_{q}\left(S^{0}\right)\right)
$$

and the isomorphism is natural for proper cellular maps.

Define $\theta_{X}$ to be the composite isomorphism

$$
E_{p, q}^{2} \rightarrow H_{p+1}\left(C_{*}^{\infty}\left(F ; h_{q}\left(S^{0}\right)\right)\right) \rightarrow{ }^{s} \tilde{H}_{p+1}\left(\dot{F} ; h_{q}\left(S^{0}\right)\right) \rightarrow{ }^{s} \tilde{H}_{p}\left(X ; h_{q}\left(S^{0}\right)\right) .
$$

Now check that $\theta$ is natural for maps: if $f: X \rightarrow Y$ then the proof of Proposition (3.4) implies that the diagram

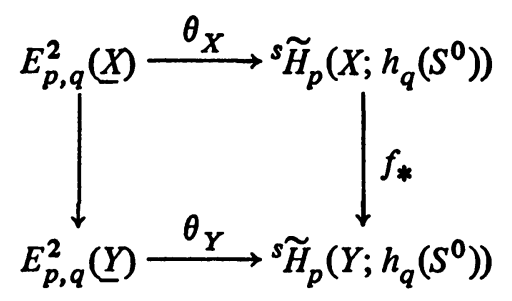

commutes.

REMARK (3.8). If $\eta: h_{*} \rightarrow h_{*}^{\prime}$ is a natural transformation of Steenrod homology theories then the diagram

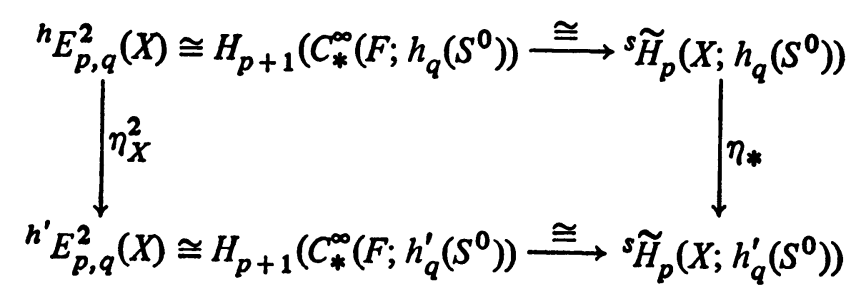

commutes, so $\theta_{X}$ is natural for transformations of theories as well. If in addition there is a short exact sequence 


$$
0 \rightarrow h_{q}\left(S^{0}\right) \stackrel{\eta_{S^{0}}}{\longrightarrow} h_{q}^{\prime}\left(S^{0}\right) \rightarrow G \rightarrow 0
$$

then there is a long exact sequence

$$
\rightarrow{ }^{h} E_{p, q}^{2}(X) \stackrel{\eta}{\rightarrow}{ }^{h} E_{p, q}^{2}(X) \rightarrow{ }^{s} \tilde{H}_{p-1}(X ; G) \rightarrow{ }^{h} E_{p-1, q}^{2} \rightarrow \cdots
$$

which is useful in studying the Chern character. In particular, there is a long exact sequence in Steenrod homology corresponding to a short exact sequence of coefficient groups.

The main theorem of this section can now be stated.

TheOREM (3.10) = TheOREM A. Let $h_{*}$ be a generalized Steenrod homology theory and let $X$ be a compact metric space of finite dimension $d$. Then there is a first quadrant spectral sequence of groups converging to $h_{*}(X)$ with $E_{p, q}^{2}$ $={ }^{s} \tilde{H}_{p}\left(X ; h_{q}\left(S^{0}\right)\right)$. The spectral sequence is natural in $X$ and in generalized Steenrod homology theories $h_{*}$. Furthermore, $E^{d+1}=E^{\infty}$.

Proof. By the previous results in this section, it remains only to prove that, for fixed $h_{*}$ and $X$, the spectral sequence is independent (from $E^{2}$ on) of the choice of CNS. So let $\underline{X}, \underline{X}^{\prime}$ be two CNS's for $X$. Then there is the usual map

$$
\text { g: }(T, F, X) \rightarrow\left(T^{\prime}, F^{\prime}, X\right)
$$

with $9 \mid X=1_{X}$ and $9 \mid F: F \rightarrow F^{\prime}$ proper and cellular. This induces a morphism of spectral sequences

$$
g_{r}: E^{r} \rightarrow E^{r}
$$

and on the $E^{2}$ level the diagram

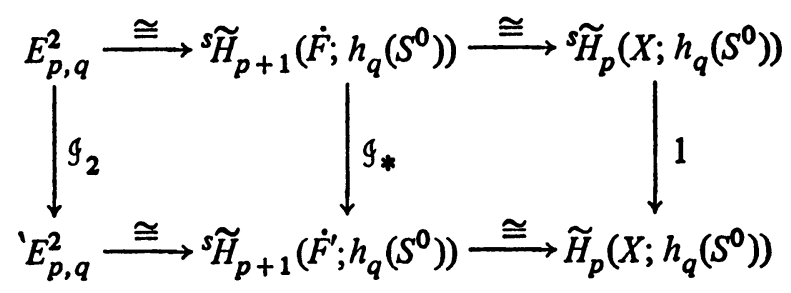

commutes. Thus $g_{2}$ is a canonical isomorphism $E^{2} \rightarrow E^{2}$, and the two spectral sequences may be identified.

RemarK (3.11). An example of D. S. Kahn [30] (see also J. Taylor [51]) shows that the spectral sequence

$$
{ }^{s} \tilde{H}_{*}\left(X ; \tilde{K}_{q}\left(S^{0}\right)\right) \Rightarrow \mathcal{E}_{*}(X)
$$


need not converge in general. He constructs an example of an infinitedimensional compact metric space $X$ with $\check{H}^{*}(X)=0$ (hence ${ }^{s} \tilde{H}_{*}(X)=0$ by the Universal Coefficient Theorem $(0.4)$ ) but $\tilde{K}^{1}(X)=\mathbf{Z} / 3$ (hence $\varepsilon_{*}(X)$ $\neq 0$ by the Universal Coefficient Theorem (0.5)). In that case $E^{2} \equiv 0$ so it has no chance of detecting the nonzero elements of $\mathcal{E}_{*}(X)$.

4. BDF homology. The Brown-Douglas-Fillmore theory revolves about a functor $\mathscr{E x t}(X)$, which we now describe. The following $C^{*}$-algebras are required: $C(X)$, the continuous complex-valued functions on a compact metric space $X$; $\mathscr{\rho}$, the bounded operators on an infinite-dimensional separable Hilbert space; $\mathscr{K} \subset \mathcal{E}$ the compact operators; and $\mathcal{E} / \mathscr{K}=\mathfrak{A}$, the Calkin algebra. An extension is a short exact sequence of $C^{*}$-algebras and $C^{*}$-algebra morphisms of the form $0 \rightarrow \mathscr{K} \rightarrow \mathcal{E} \rightarrow C(X) \rightarrow 0$ where $\mathscr{E}$ is a $C^{*}$-algebra containing $\mathcal{K}$ and $I$ (the identity operator) and contained in $\mathcal{L}$. This data produces the important diagram

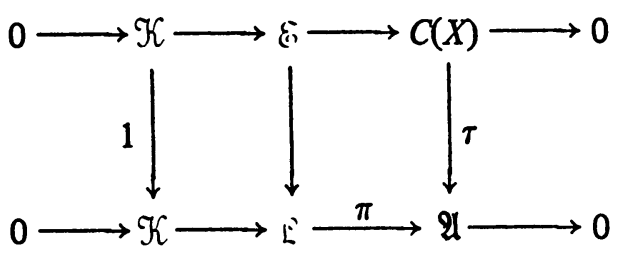

illustrating that an extension may also be viewed as a $C^{*}$ - injection $\tau: C(X) \rightarrow \mathfrak{A}$. Two extensions $\tau$ and $\tau^{\prime}$ are equivalent if there is a unitary $u \in \mathfrak{A}$ such that $\tau^{\prime}(f)=u^{*} \tau(f) u$ for all $f \in C(X)$.

If $\tau_{1}, \tau_{2}: C(X) \rightarrow \mathfrak{U}$ are extensions, then their sum $\tau_{1} \oplus \tau_{2}$ is defined to be the composite

$$
C(X) \stackrel{\left(\eta, \tau_{2}\right)}{\longrightarrow} \mathfrak{A}_{1} \oplus \mathfrak{A}_{2} \hookrightarrow \mathfrak{A} \otimes M_{2} \cong \mathfrak{A}
$$

where $M_{2}$ is the $2 \times 2$ matrix ring. The isomorphism $\mathfrak{A} \otimes M_{2} \cong \mathfrak{A}$ is unique up to unitary equivalence. With this definition of addition, unitary equivalence classes of extensions form an abelian group, denoted by $\mathcal{E x t}(X)$. (The identity under addition may be represented by any extension $\tau: C(X) \rightarrow \mathfrak{A}$ which factors through $\&$ It is not trivial to exhibit additive inverses.)

Suppose that $f: X \rightarrow Y$ is a continuous surjection. Then the correspondence

$$
C(X) \stackrel{\leftarrow}{\longrightarrow} \mathfrak{A} \mapsto C(Y) \stackrel{f^{*}}{\longrightarrow} C(X) \stackrel{\Im}{\longrightarrow} \mathfrak{A}
$$

induces a homomorphism of abelian groups

$$
f_{*}: \operatorname{Ext}(X) \rightarrow \mathcal{E x t}(Y) \text {. }
$$


In fact the same idea with a simple modification works for any continuous function $f$.

PROPOSITION (4.2) (BDF [15], [ 16]). Ext is a covariant functor from compact metric spaces to abelian groups. Further, Ext is homotopy invariant-if $f_{0} \simeq f_{1}: X$ $\rightarrow Y$ then

$$
f_{0 *}=f_{l_{*}}: \delta x t(X) \rightarrow \delta x t(Y) .
$$

It follows that if $X$ and $Y$ are homotopy equivalent spaces, then $\operatorname{Ext}(X)$ $\cong \operatorname{Ext}(Y)$. (For example, the circle $S^{1}$ and the annulus $S^{1} \times\left[\frac{1}{2}, 1\right]$ are homotopy equivalent.)

The functor $\delta x t$ was devised by BDF to study several sorts of problems in operator theory.

Problem 1. Given an essentially normal operator $T \in \mathcal{L}$ (i.e., an operator $T$ with $\left.T^{*} T-T T^{*} \in \mathfrak{F}\right)$, when is $T$ of the form (normal) + (compact)?

Solution (BDF). Any operator of the form $N+K=T$ has ind $(T-\lambda I)$ $=0$ for all $\lambda \notin \sigma(\pi T)=\sigma_{e}(T)$ (the essential spectrum). This condition is sufficient.

Problem 2. When are two essentially normal operators $T_{0}, T_{1}$ unitarily equivalent modulo compacts?

Solution (BDF). If $T_{1}=U^{*} T_{0} U+K$ then $\sigma_{e}\left(T_{1}\right)=\sigma_{e}\left(T_{0}\right)=X$ and

$$
\operatorname{ind}\left(T_{0}-\lambda I\right)=\operatorname{ind}\left(T_{1}-\lambda I\right)
$$

for all $\lambda \notin X$. These two conditions suffice.

In both of these cases the relevant obstruction group $8 \mathrm{xt}(X)$ which arises has $X \subset \mathbf{C}$. So restrict to that case temporarily. Here is one way to produce elements of $\operatorname{Ext}(X)$. Pick an essentially normal operator $T$ with $\sigma_{e}(T)=X$. Let $\mathcal{E}_{T}$ be the $C^{*}$-algebra in $\mathcal{E}$ generated by the identity $I, T$, and $\mathcal{K}_{0}$ Then the short exact sequence

$$
0 \rightarrow \nVdash \rightarrow \varepsilon_{T} \rightarrow \varepsilon_{T} / \mathcal{K} \cong C(X) \rightarrow 0
$$

yields an element $[T] \in \mathcal{E} \mathrm{xt}(X)$. In fact every element of $\mathcal{E x t}(X)$ arises in this fashion (for $X \subset \mathbf{C}$ !).

Following BDF, let us now describe $\operatorname{Ext}(X)$ for $X \subset \mathbf{C}$ (from which follows the solutions to Problems 1 and 2 above).

Recall first that an operator $T \in \mathcal{L}$ is Fredholm if it has closed range and $\operatorname{ker} T$ and $\operatorname{ker} T^{*}$ are finite dimensional. In that case define the Fredholm index of $T$ to be

$$
\operatorname{index}(T)=\operatorname{dim} \operatorname{ker} T-\operatorname{dim} \operatorname{ker} T^{*} .
$$


By Atkinson's theorem, $T$ is Fredholm if and only if $\pi T$ is invertible in $\mathscr{A}$.

Let $\tau \in \operatorname{Ext}(X)$. Then $\tau$ may be represented as $\tau: C(X) \rightarrow \mathfrak{A}$. Restrict $\tau$ to those functions in $C(X)$ which never assume the value 0 . Then $\tau: C(X)^{\circ} \rightarrow \mathfrak{A}^{\circ}$ (where $\Lambda$ is the group of invertible elements in the ring $\Lambda$ ). The Fredholm index may be regarded as a continuous homomorphism of groups ind: $\mathfrak{A}^{*}$ $\rightarrow \mathbf{Z}$. Composing yields

$$
\text { ind } \circ \tau: C(X)^{\cdot} \rightarrow \mathbf{Z}
$$

which is continuous, respects homotopies and induces a map

$$
\gamma_{1}(\tau): \pi^{1}(X) \rightarrow \mathbf{Z}
$$

Here $\pi^{1}(X)$ is the group of based homotopy classes of maps from $X$ to $S^{1}$. One checks that in fact $\gamma_{1}$ is a homomorphism

$$
\gamma_{1}: \operatorname{\delta xt}(X) \rightarrow \operatorname{hom}\left(\pi_{1}(X), \mathbf{Z}\right) .
$$

An easy topological argument shows that $\operatorname{hom}\left(\pi^{1}(X), \mathbf{Z}\right) \cong \tilde{H}^{0}(\mathbf{C}-X)$ where $\tilde{H}^{0}(\mathbf{C}-X)$ denotes homotopy classes of maps from the bounded components of $\mathbf{C}-X$ to $\mathbf{Z}$; i.e., locally constant integer-valued functions on the bounded components of $\mathbf{C}-X$. Let $\gamma: \delta x t(X) \rightarrow \tilde{H}^{0}(\mathbf{C}-X)$ be the composite.

THEOREM (4.3) (BDF) . Let $X$ be a compact subset of C. Then $\gamma: \mathcal{E x t}(X)$ $\rightarrow \tilde{H}^{0}(\mathbf{C}-X)$ is an isomorphism of groups.

It is easy to describe $\gamma$ explicitly in terms of the classes $[T] \in \mathcal{E x t}(X)$ - the function $\gamma[T]$ is that function which assigns to $\lambda \in \mathbf{C}-X$ the Fredholm index of $T-\lambda I$. Thus $\gamma[T]=0$ if and only if ind $(T-\lambda I)=0$ for all $\lambda$ for which it is defined.

An extension $0 \rightarrow \mathscr{K} \rightarrow \mathcal{E} \stackrel{\pi}{\rightarrow} C(X) \rightarrow 0$ represents the zero element of $\mathcal{E x t}(X)$ if and only if there is a $C^{*}$-algebra morphism $\sigma: C(X) \rightarrow \mathcal{E}$ which is a section; $\pi \sigma=1$. In the case at hand, $[T]=0$ means $\pi T=\pi \sigma \pi T$, so $\pi(T-\sigma \pi T)=0$, implying $T=\sigma \pi T+K$ where $K$ is compact and $\sigma \pi T$ is normal. Thus $T$ is (normal) + (compact) if and only if ind $(T-\lambda I)=0$ where defined, vindicating the solution recorded above to Problem 1. The solution to Problem 2 is similar.

Remark (4.4). It seems plausible that there may be an "operator theoretic" proof of Theorem (4.3), and hence an "operator theoretic" solution to the operator-theory problems which originally motivated BDF. But for $X \llbracket \mathbf{C}$ the situation is much more complicated topologically. Special cases of (4.3) do have "operator theoretic" proofs-cf. Douglas [18] $\left(X=S^{1}\right)$, DeddensStampfli [17] $\left(X=S^{1} \times I\right)$, Behncke-Leptin [10] $(X \subset \mathbf{R})$. 
5. BDF homology and $K$-theory. For $X \subset \mathrm{C}$, Theorem (4.3) gives a very satisfying description of $\operatorname{Ext}(X)$. But of greater interest to topologists is the behavior of $\operatorname{Ext}(X)$ for higher dimensional spaces. The key result is Theorem (5.1). (Recall that $S^{2} X$ is the second unreduced suspension of $X$.)

THeOREM (5.1) (BDF). Let $X$ be compact metric. Then there is a natural isomorphism $\delta \mathrm{xt}\left(S^{2} X\right) \cong \varepsilon \mathrm{xt}(X)$.

Let us refer to this as the Bott periodicity isomorphism, since $E x t$ is related to $K$-theory (as will be indicated). Define groups $\varepsilon_{n}(X)$ for $n \in Z$ by

$$
\varepsilon_{n}(X)= \begin{cases}\varepsilon x \mathrm{t}(X), & \text { if } n \text { is even. } \\ \varepsilon \mathrm{xt}(S X), & \end{cases}
$$

THEOREM (5.2) (BDF). $\mathcal{E}_{*}$ is a generalized Steenrod homology theory on the category of compact metric spaces. The coefficient groups are given by

$$
\mathcal{E}_{n}\left(S^{0}\right)= \begin{cases}\mathbf{Z}, & \text { if } n \text { is even, } \\ 0, & \text { if } n \text { is odd } .\end{cases}
$$

Note that $\varepsilon_{*}$ is not additive $\left(\varepsilon_{*}\left(\bigvee_{j} X_{j}\right) \neq \oplus_{j} \mathcal{E}_{*}\left(X_{j}\right)\right)$ and is not a "representable" homology theory on the category of compact metric spaces. On the (smaller) category of finite complexes $\mathcal{E}_{*}$ is representable; it is naturally equivalent to $\tilde{K}_{*}$ (see Theorem (5.4)).

Remarks on the ProOf of (5.2). The theory $\varepsilon_{*}$ satisfies the suspension axiom (S) by fiat and Theorem (5.1). Axioms (E) (exact sequence) and (W) (wedge) are proven for $\varepsilon_{x t}$ in [15] and this easily extends to $\mathscr{E}_{*}$. The computation of $\mathcal{E}_{n}\left(S^{0}\right)=\varepsilon \times \mathrm{xt}\left(S^{n-1}\right)$ is basic. The group $\varepsilon_{\mathrm{xt}}\left(S^{1}\right)$ is isomorphic to $\tilde{H}^{0}\left(\mathrm{C}-S^{1}\right)=\mathbf{Z}$ and the group $\operatorname{Ext}\left(S^{0}\right)$ is isomorphic to $\tilde{H}^{0}\left(\mathrm{C}-S^{0}\right)=0$ (4.3).

Given Theorem (5.2), it is natural to inquire whether or not $\varepsilon_{*}$ has previously been studied by topologists. The best known homology theory of period 2 is complex homology $K$-theory. BDF prove that $\varepsilon_{*} \cong \tilde{K}_{*}$ on finite complexes. We wish to capitalize upon this theorem-this requires a short digression into $K$-theory [2], [6], [19], [27].

The cohomology theory $K^{*}$ is defined by means of vector bundles. For some purposes, however, the following "analytic" description (due to Atiyah and Jänich [6], [28]) is more convenient. Since $K^{n}=K^{n+2}$ it suffices to define $K^{0}$ and $K^{1}$.)

$$
K^{0}(X)=[X, \mathscr{F}], \quad K^{1}(X)=[X, \text { Q }],
$$

where $\mathscr{F}$ is the space of Fredholm operators in $\mathcal{L}$ (with the norm topology), $\mathcal{Q}=$ inj $\lim \mho(n)$ the infinite unitary group, and $[X, Y]$ denotes homotopy 
classes of maps from $X$ to $Y$. Note also that $\mathscr{F} \simeq \mathfrak{U}^{*}$, the invertibles in the Calkin algebra. Then $K^{*}$ is a generalized cohomology theory. The map (point) $\rightarrow X$ induces a split exact sequence

$$
0 \rightarrow \tilde{K}^{*}(X) \rightarrow K^{*}(X) \rightarrow K^{*} \text { (point) } \rightarrow 0
$$

and

$$
K^{n} \text { (point) }=\tilde{K}\left(S^{0}\right)= \begin{cases}\mathbf{Z}, & \text { if } n \text { is even, } \\ 0, & \text { if } n \text { is odd. }\end{cases}
$$

There is a basic connection between $\varepsilon_{*}$ and $K^{*}$ due to BDF. Define $\gamma_{\infty}: \operatorname{Ext}(X) \rightarrow \operatorname{hom}\left(K^{1}(X), \mathbf{Z}\right)$ as follows: let $\tau: C X \rightarrow \mathfrak{A}$ and $[f] \in K^{1}(X)$ be represented by $f: X \rightarrow$ Q $(n)$ (such an $n$ exists since $X$ is compact). Let

$$
\gamma_{\infty}(\tau)(f)=\text { ind }((\tau \otimes 1) f)
$$

where $\tau \otimes 1: C(X) \otimes M_{n} \rightarrow \mathfrak{U} \otimes M_{n} \cong \mathfrak{U}$. The map $\gamma_{\infty}$ is well defined and is a natural generalization of $\gamma$. (This may be made more explicit by observing that $K^{1}(X) \cong \pi^{1}(X)$ if $X \subset \mathrm{C}$.) The map $\gamma_{\infty}$ should be thought of as a Kronecker pairing, analogous to the more familiar maps $H_{n}(X)$ $\rightarrow \operatorname{hom}\left(H^{n}(X), \mathbf{Z}\right)$ for ordinary homology.

Given any cohomology theory defined on finite complexes, there is an associated homology theory. This was first systematized by George Whitehead [53] using spectra. He showed, moreover, that Spanier-Whitehead duality could be used to pass from a cohomology theory to a homology theory. This is the appropriate point of view in our context.

Suppose $X$ is a finite simplicial complex embedded in $S^{n+1}$. An $n$-dual of $X$ is a finite simplicial complex $D_{n} X \subset S^{n+1}-X$ which is a deformation retract of $S^{n+1}-X$. Such duals exist, for given a complex $X$ embedded linearly in some triangulation $K$ of $S^{n+1}$, the supplement of $X$ in the first derived of $K$ is an $n$-dual of $X$. Moreover, any two duals $D_{n} X$ and $D_{m} X$ are of the same stable homotopy type. Taking duals is essentially unique in the sense that $X$ is an $n$ dual of $D_{n} X$. Taking duals "commutes" with suspension in the sense that $\underline{S} D_{n} X$ is an $(n+1)$-dual of $X$ and $D_{n+1} \underline{S} X$ is an $n$-dual of $X$. (Recall that $\underline{S} X=S^{1} \wedge X$ denotes reduced suspension.)

Homology $K$-theory is defined by

$$
\tilde{K}_{j}(X)=\tilde{K}^{-j}\left(D_{2 n} X\right) \text {. }
$$

It can be shown that $K_{*}$ is a generalized homology theory on finite complexes [53]. There is more than one reasonable definition of $K_{*}(X)$ for more general spaces $X$; one aim of this paper is to argue for $\varepsilon_{*}$ as being the appropriate extension of $K_{*}$ to compact metric spaces. 
THEOREM (5.4) (BDF) [55]. On the category of finite complexes there is a natural equivalence of homology theories $\mathcal{E}_{*} \cong \tilde{K}_{*}$.

One first needs to construct a natural transformation of homology theories $\Gamma_{X}: \varepsilon_{*}(X) \rightarrow \tilde{K}_{*}(X)$ such that $\Gamma_{X}$ is an isomorphism when $X=S^{n}$, all $n$. (The argument is then completed by induction or by appeal to a spectral sequence.) The map $\Gamma_{X}$ is constructed as follows.

If $X \subset S^{2 n+1}$ then there is a canonical duality pairing [47], [48]

$$
X \wedge D_{2 n} X \rightarrow S^{2 n}
$$

and hence a canonical map

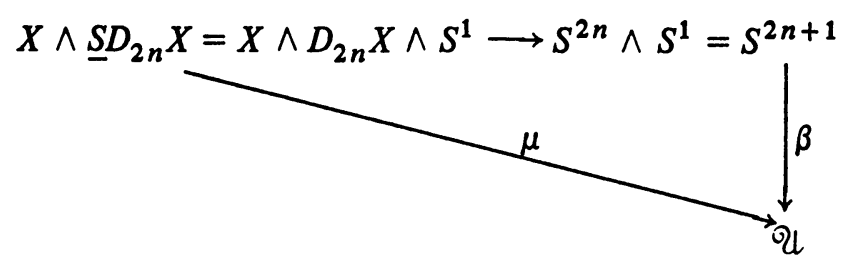

where $[\beta] \in \pi_{2 n+1}($ (Q) $=\mathbf{Z}$ represents the Bott generator [19]. Then [ $\mu$ ] $\in\left[X \wedge \underline{S} D_{2 n} X, Q\right]_{0}$ (where $[X, Y]_{0}$ is based homotopy classes of basepointpreserving maps). The map $\Gamma_{X}: \operatorname{Ext}(X) \rightarrow \tilde{K}_{1}(X)$ is to be the composite

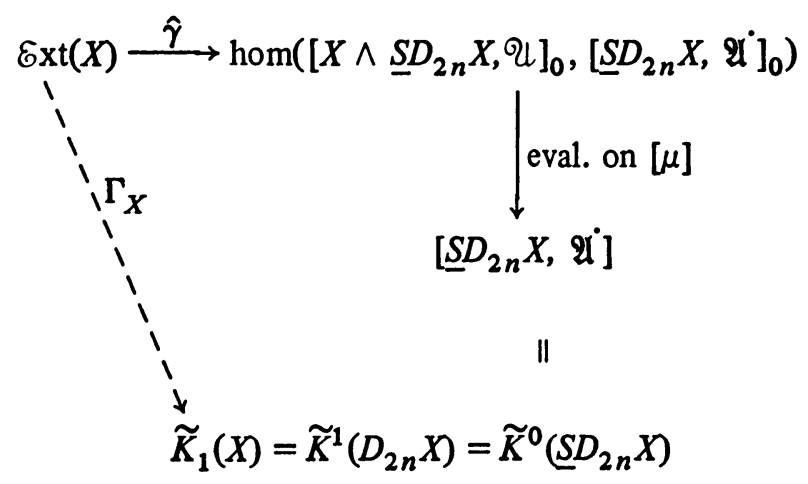

and it remains to define $\hat{\gamma}$. The map $\hat{\gamma}$ is a special case (with $Y=\underline{S} D_{2 n} X$ ) of the map

$$
\gamma_{Y}: \operatorname{Ext}(X) \rightarrow \operatorname{hom}\left([X \wedge Y, \mathscr{Q}]_{0},\left[Y, \mathfrak{I}^{*}\right]_{0}\right)
$$

which is defined as follows.

Let $X$ and $Y$ be finite complexes and $\tau \in \mathcal{E} \mathrm{xt}(X)$. Represent $\tau$ as $\tau: C(X) \rightarrow \mathfrak{U}$. Let $f: X \wedge Y \rightarrow$ थ represent an element of $[X \wedge Y, थ]_{0}$. The 
map $f$ factors through some $थ(n)$. Let $f: Y \rightarrow \mathcal{Q}(n)^{X}$ be the adjoint. Then $\tilde{f}(y)=\left(\tilde{f}_{i, j}(y)\right)$, is an $n \times n$ matrix of complex-valued functions on $X$. Applying $\tau$ to each term gives $\tau(\tilde{f}(y))=\left(\tau \tilde{f}_{i j}(y)\right)$, an invertible element of $\mathfrak{A} \otimes M_{n} \cong \mathfrak{U}$. The correspondence $y \mapsto \tau(\tilde{f}(y))$ defines a continuous function $Y \rightarrow \mathfrak{U}$ which is a representative of $\gamma_{Y}(\tau)$.

Now bring the results of $\S 3$ to bear on $\varepsilon_{*}$. For convenience we restate (in specialized form) the main theorems.

TheOREM (5.6). Let $X$ be compact metric of dimension $d<\infty$. Then there is a spectral sequence which is natural in $X$, converges to $\mathcal{E}_{*}(X)$, has $E^{d+1}=E^{\infty}$, and

$$
E_{p, q}^{2}={ }^{s} \tilde{H}_{p}\left(X ; \tilde{K}_{q}\left(S^{0}\right)\right) \cong \begin{cases}{ }^{s} \tilde{H}_{p}(X), & q \text { even } \\ 0, & \text { q odd }\end{cases}
$$

Note that the even differentials $d_{2 r}$ are identically zero, since $E_{p, q}^{r}=0$ for $q$ odd. The first potentially nonzero differential is thus $d_{3}$.

THEOREM (5.7). The $\lim ^{1}$ sequence

$$
0 \rightarrow \mathfrak{L} \tilde{K}_{n}(X) \rightarrow \varepsilon_{n}(X) \rightarrow \check{K}_{n}(X) \rightarrow 0
$$

is exact and natural for maps of spaces.

The third main computational tool is the Universal Coefficient Theorem for $\mathscr{E}_{*}$, denoted UCT. This theorem was proved for finite complexes by D. W. Anderson [3]. The general case is due to L. G. Brown [14]. His interesting proof uses algebraic $K$-theory to identify ker $\gamma_{\infty}$.

Theorem (5.8) (UCT) (ANDERson, Brown). Let $X$ be compact metric. Then there is a natural short exact sequence

$$
0 \rightarrow \operatorname{Ext}\left(\tilde{K}^{0}(X), \mathbf{Z}\right) \rightarrow \operatorname{Ext}(X) \stackrel{\gamma_{\infty}}{\rightarrow} \operatorname{hom}\left(\tilde{K}^{1}(X), \mathbf{Z}\right) \rightarrow 0
$$

which (unnaturally) splits (and similarly for $\mathcal{E}_{n}(X)$ ).

REMARK (5.9). The "Ext" appearing in the statement is from homological algebra: Ext $(A, B)$ is equivalence classes of short exact sequences of abelian groups of the form $0 \rightarrow B \rightarrow G \rightarrow A \rightarrow 0$. For example, if $\tilde{K}^{0}(X)$ is a free abelian group then $\operatorname{Ext}\left(\tilde{K}^{0}(X), \mathbf{Z}\right)=0$ and $\gamma_{\infty}$ is an isomorphism.

Theorem (5.8) implies

Corollary (5.10). Let $X$ and $Y$ be compact metric spaces. If $X$ and $Y$ have the same shape then $\mathcal{E}_{*}(X) \cong \mathcal{E}_{*}(Y)$. 
Proof. Since $K^{0}(X)=[X, \mathscr{F}]$ and $K^{\prime}(X)=[X, \mathcal{Q}]$ and $\mathscr{F}$ and $\mathcal{Q}$ are ANR's, the functors $K^{0}$ and $K^{1}$ are shape-invariant [32]. Apply the split exact sequence (5.8) to obtain $\varepsilon_{*}(X) \cong \varepsilon_{*}(Y)$.

6. Some calculations of $\varepsilon_{*}(X)$. In this section $\varepsilon_{*}(X)$ is computed in various cases. Some cases involve translating known results on $K^{*}(X)$ via Theorem (5.8). The main reference is Atiyah's book. The presentation of these facts is intended to give analysts a feeling for which groups are associated to which spaces. The later cases require techniques which use properties of $\mathcal{E}_{*}$ itself.

Proposition (6.1) ([6, p. 80]). Let $X$ be a finite cell complex such that $X^{(2 k)}=X^{(2 k+1)}$ for all $k$ (e.g., $\mathbf{C} P^{n}$, complex grassmanns, flag manifolds, complex quadrics). Then $\varepsilon_{1}(X)=0$ and $\varepsilon_{0}(X)=\mathbf{Z}^{r-1}$ where $r$ is the number of cells of $X$.

PROPOSITION (6.2) ([6, p. 107]). The groups $\varepsilon_{*}\left(R P^{k}\right)$ are given by $\varepsilon_{0}\left(R P^{k}\right)$ $=0$ and

$$
\mathcal{E}_{1}\left(R P^{2 n}\right)=\mathbf{Z} / 2^{n}, \quad \mathcal{E}_{1}\left(R P^{2 n+1}\right)=\mathbf{Z} \oplus \mathbf{Z} / 2^{n} .
$$

Proposirion $(6.3)\left(\left[6\right.\right.$, p. 116]). The groups $\mathcal{E}_{*}(น(n))$ are given by

$$
\mathcal{E}_{1}(U(n))=\mathbf{Z}^{2 n-1} \text { and } \mathscr{E}_{0}(\mathcal{Q}(n))=\mathbf{Z}^{2^{n-1}-1} \text {. }
$$

Remark (6.4). Atiyah shows that $K^{*}(U(n))$ is an exterior algebra on the $n$ exterior power representations of $\mathcal{Q}(n)$. More generally, Hodgkin [26] has shown that if $G$ is a compact, connected, simply connected Lie group then $K^{*}(G)$ is an exterior algebra on certain representations of $G$. Thus $\varepsilon_{*}(G)$ is free abelian.

REMARK (6.5). The lens space $L^{n}(p)=S^{2 n+1} / p$ has $\mathscr{E}_{1}\left(L^{n}(p)\right)=\mathbf{Z}$ $\oplus\left(\mathbf{Z} / p^{s+1}\right)^{r} \oplus\left(\mathbf{Z} / p^{s}\right)^{p-r-1}$, where $n=s(p-1)+r$ and $0 \leqslant r<p-1$; thus $\varepsilon_{1}\left(L^{n}(p)\right)$ has a $p$-group summand.

The second class of spaces we shall consider are those for which $\lim ^{1}$ techniques are appropriate. The classical examples are the solenoids $\Sigma_{G}$ described in $\S 1$.

Proposition (6.6). The $\varepsilon_{*}$-groups of the dyadic solenoid $\Sigma_{2}$ are given by $\mathcal{E}_{1}\left(\Sigma_{2}\right)=0$ and $\mathscr{E}_{0}\left(\Sigma_{2}\right)=\hat{\mathbf{Z}}_{2} / \mathbf{Z}$.

Proof. The $\lim ^{1}$ sequences read

$$
\begin{aligned}
& \underset{\substack{\| \\
0}}{\mathcal{L} \tilde{E}_{1}\left(\Sigma_{2}\right)} \rightarrow \varepsilon_{1}\left(\Sigma_{2}\right) \rightarrow \check{\mathscr{E}}_{1}\left(\Sigma_{2}\right) \rightarrow 0, \\
& 0 \rightarrow \mathfrak{L} \varepsilon_{0}\left(\Sigma_{2}\right) \rightarrow \varepsilon_{0}\left(\Sigma_{2}\right) \rightarrow \check{\varepsilon}_{1}\left(\Sigma_{2}\right) \rightarrow 0 .
\end{aligned}
$$


Further, $\check{\zeta}_{1}\left(\Sigma_{2}\right)=$ proj $\lim (\mathbf{Z}, 2)=0$, and

$$
\left\llcorner\mathcal{E}_{0}\left(\Sigma_{2}\right)=\operatorname{proj} \lim ^{1}(Z, 2)=\hat{\mathbf{Z}}_{2} / \mathbf{Z}\right.
$$

by the proof of (1.9).

REMARK (6.7). Proposition (6.6) also follows from the spectral sequence and (1.9), or alternatively from the UCT using the fact that $K^{1}\left(\Sigma_{2}\right)=\mathbf{Z}\left[\frac{1}{2}\right]$.

The third type of spaces we consider are those which embed in lowdimensional Euclidean space.

THEOREM (6.8). Suppose that $X$ is a finite connected complex in $\mathbf{R}^{5}$. Then the spectral sequence converging to $\varepsilon_{*}(X)$ has $E^{2}=E^{\infty}$ and there is a natural isomorphism

$$
\mathcal{E x t}(X) \cong{ }^{s} \tilde{H}_{1}(X) \oplus{ }^{s} \tilde{H}_{3}(X)
$$

Proof. The BDF identification of $\varepsilon_{*}$ with $K_{*}$, Spanier-Whitehead duality and simplicial approximation together imply that

$$
\operatorname{Ext}(X) \cong\left[D_{4} X, थ(3)\right]
$$

where $थ(3)$ is the group of $3 \times 3$ unitary matrices. The fibration $थ(2) \rightarrow U(3)$ $\rightarrow S^{5}$ induces an exact sequence

$$
\left[D_{4} X, \Omega S^{5}\right] \rightarrow\left[D_{4} X, \text { थ(2) }\right] \rightarrow\left[D_{4} X, \text { थ(3) }\right] \rightarrow\left[D_{4} X, S^{5}\right] \text {. }
$$

Since $D_{4} X$ is a finite complex in $\mathbf{R}^{5},\left[D_{4} X, S^{5}\right]=\tilde{H}^{5}\left(D_{4} X\right)=0$. Similarly the group $\left[D_{4} X, \Omega S^{5}\right]=0$, since

$$
\left[D_{4} X, \Omega S^{5}\right]=\left[\underline{S} D_{4} X, S^{5}\right]=\tilde{H}^{5}\left(\underline{S} D_{4} X\right)
$$

by the Steenrod classification theorem $[47$, p. 460$]$ and

$$
\tilde{H}^{5}\left(\underline{S} D_{4} X\right)=\tilde{H}^{4}\left(D_{4} X\right)=\tilde{H}_{0}(X)=0
$$

since $X$ is connected. Then (6.10) implies that $\operatorname{Ext}(X) \cong\left[D_{4} X, \mathcal{Q}(2)\right]$. But $थ(2)$ is topologically equivalent to $S^{1} \times S^{3}$. Assembling this information yields

$$
\operatorname{Ext}(X) \cong\left[D_{4} X, \mathcal{Q}(2)\right] \cong\left[D_{4} X, S^{1}\right] \oplus\left[D_{4} X, S^{3}\right] .
$$

But the natural map $\left[D_{4} X, S^{3}\right] \rightarrow \tilde{H}^{3}\left(D_{4} X\right)$ is an isomorphism since $\tilde{H}^{5}\left(D_{4} X\right)=0$ and $\tilde{H}^{4}\left(D_{4} X ; \mathbf{Z} / 2\right)=0$; hence

$$
\delta x t(X) \cong \tilde{H}^{-1}\left(D_{4} X\right) \oplus \tilde{H}^{3}\left(D_{4} X\right) \cong \tilde{H}_{3}(X) \oplus \tilde{H}_{1}(X)
$$


as claimed, and all isomorphisms are natural. This forces the differential $d_{3}: \tilde{H}_{4}(X) \rightarrow \tilde{H}_{1}(X)$ to be trivial, so $E^{2}=E^{\infty}$, completing the proof.

THEOREM (6.12) (THeOREM B). Suppose $X$ is a compact subspace of $(\mathbf{R})^{4}$. Then the spectral sequence converging to $\mathcal{E}_{*}(X)$ has $E^{2}=E^{\infty}$ and the groups $\mathcal{E}_{*}(X)$ are given by the exact sequences

$$
\begin{aligned}
& 0 \rightarrow{ }^{s} \tilde{H}_{1}(X) \rightarrow \varepsilon_{1}(X) \rightarrow{ }^{s} \tilde{H}_{3}(X) \rightarrow 0, \\
& 0 \rightarrow{ }^{s} \tilde{H}_{0}(X) \rightarrow \varepsilon_{0}(X) \rightarrow{ }^{s} \tilde{H}_{2}(X) \rightarrow 0 .
\end{aligned}
$$

The sequence (6.12) naturally splits, if $X$ is a finite complex, and it unnaturally splits in general.

Proof. Suppose first that $X$ is a finite complex contained in $(\mathbf{R})^{4}$. Then $S X$ is a finite connected complex contained in $(\mathbf{R})^{5}$, so Theorem (6.8) applies. Observe that $\varepsilon_{n}(S X)=\varepsilon_{n+1}(X)$. Then Tueorem (6.11) holds for finite complexes, since (6.12) and (6.13) follow directly from the collapse of the spectral sequence.

In the general case, the spectral sequence (Theorem $A$ ) converging to $\varepsilon_{*}(X)$ has $E_{p, q}^{2}=0$ unless $q$ is even and $p=0,1,2,3$. Then there is an exact sequence $0 \rightarrow{ }^{s} \tilde{H}_{1} X \rightarrow \varepsilon_{1}(X) \rightarrow \operatorname{ker} d_{3} \rightarrow 0$ where $\operatorname{ker}\left(d_{3}\right) \subset{ }^{s} \tilde{H}_{3}(X)$. It thus suffices to prove that $\varepsilon_{1}(X)$ maps onto $\tilde{H}_{3}(X)$ to complete the proof.

Let $X=$ proj $\lim X_{j}$ as usual. Apply (6.9) and take inverse limits to obtain $\check{\mathscr{E}}_{1} X=\check{H}_{1}(X) \oplus \check{H}_{3}(X)$. But note that $\mathfrak{e}^{s} H_{3}(X)=0$, so $\check{H}_{3}(X) \cong{ }^{s} \tilde{H}_{3}(X)$. Thus each of the maps

$$
\varepsilon_{1}(X) \rightarrow \check{\varepsilon}_{1}(X) \rightarrow \check{H}_{3}(X)={ }^{s} \tilde{H}_{3}(X)
$$

is onto, so $\operatorname{ker} d_{3}={ }^{s} \tilde{H}_{3}(X)$.

To complete the proof it suffices to show that (6.12) splits. But consider the diagram

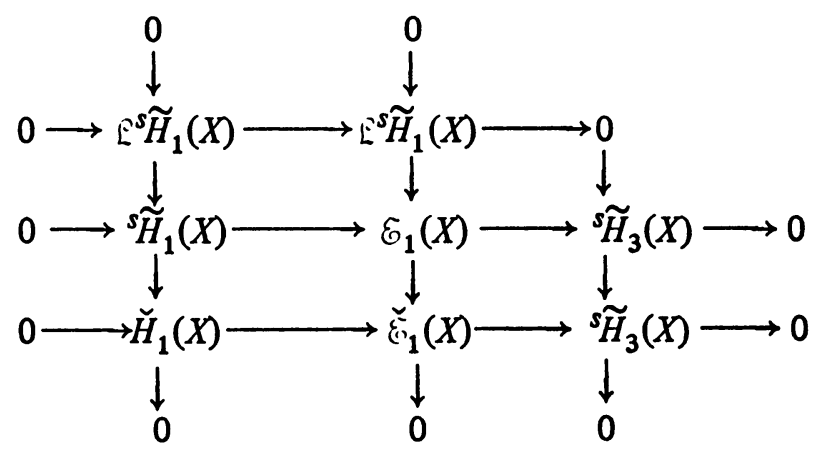

Thus 


$$
\begin{aligned}
\mathcal{E}_{1}(X) & =\check{E}_{1}(X) \oplus \mathcal{L}^{s} \tilde{H}_{1}(X) \quad \text { (unnaturally) } \\
& =\check{H}_{1}(X) \oplus{ }^{s} \tilde{H}_{3}(X) \oplus \mathcal{L}^{s} \tilde{H}_{1}(X) \\
& ={ }^{s} \tilde{H}_{1}(X) \oplus{ }^{s} \tilde{H}_{3}(X)
\end{aligned}
$$

so (6.12) splits unnaturally.

Remark (6.14). For arbitrary subsets of (R) there may possibly be nonzero $d_{3}$-differentials, corresponding to natural transformations

$$
\check{H}_{4}(X) \rightarrow \mathcal{L} \tilde{H}_{1}(X) \text { and }{ }^{s} \tilde{H}_{3}(X) \rightarrow{ }^{s} \tilde{H}_{0}(X) .
$$

REMARK (6.15). The nonzero $d_{3}$-differentials on finite complexes correspond to the cohomology operation $\beta S q^{2}[9]$.

We close this section with an application of our calculation of $\delta \mathrm{xt}(X)$ for $X \subset \mathbf{R}^{4}$ to operator theory. (The relevance of $\varepsilon_{*}$ in higher dimensions to operator theory was first noted by BDF [16, p. 119].) Suppose $A_{1}$ and $A_{2}$ are essentially normal operators such that $\pi A_{1}$ and $\pi A_{2}$ commute. When do there exist compact perturbations

$$
A_{j}=B_{j}+K_{j}, \quad j=1,2,
$$

with $B_{1}$ and $B_{2}$ commuting normals? Let $X=j t \sigma_{e}\left(A_{1}, A_{2}\right)$ be the joint spectrum of $\pi A_{1}$ and $\pi A_{2}$, let $\mathcal{E}$ be the $C^{*}$-algebra generated by $\Re, I, A_{1}, A_{2}$, and let $\tau\left[A_{1}, A_{2}\right] \in \delta \times \mathrm{t}(X)$ be the extension $0 \rightarrow \mathscr{K} \rightarrow \mathcal{E} \rightarrow C(X) \rightarrow 0$. Then $\tau\left[A_{1}, A_{2}\right]=0$ precisely when the perturbation exists. But note that $X \subset R^{4}$; hence

$$
0 \rightarrow{ }^{s} \tilde{H}_{1}(X) \rightarrow \operatorname{Ext}(X) \stackrel{\ulcorner}{\rightarrow} \tilde{H}_{3}(X) \rightarrow 0
$$

is exact. The group ${ }^{s} \tilde{H}_{1}(X)$ measures the "one-dimensional holes" in $X$ and corresponds to the usual Fredholm index. (In particular, it is obvious that $\tau\left(A_{1}, A_{2}\right)=0$ implies ind $\left(A_{1}-\lambda I\right)=0$ where defined; this information is carried by ${ }^{s} \tilde{H}_{1}(X)$.)

Proposition (6.16). Suppose that $\operatorname{Ext}\left(H^{2}(X), Z\right)=0$. If ind $\left(A_{i}-\lambda I\right)=0$ where defined and $\Gamma \tau\left[A_{1}, A_{2}\right]=0$ (e.g. if $\left.H_{3}(X)=0\right)$ then the perturbation exists.

This theme is expanded upon in [57].

REMARK (6.17). It seems to be difficult to identify $\tau\left[A_{1}, A_{2}\right]$ even if one is given $\sigma_{e}\left(A_{1}\right)$ and $\sigma_{e}\left(A_{2}\right)$. For our purposes, identify means to identify certain classes in certain homology groups. The following example indicates the twofold difficulty involved. Let $S=A_{1}+i A_{2}$ be the unilateral shift, written as the sum of its real and imaginary parts. Then 


$$
\begin{aligned}
\sigma_{e}\left(A_{1}\right) & =\sigma_{e}\left(A_{2}\right)=[0,1], \\
j t \sigma_{e}\left(A_{1}, A_{2}\right) & =\sigma_{e}(S)=S^{1},
\end{aligned}
$$

so a new "hole" has been created. But the situation is even worse. The operators $A_{1}$ and $A_{2}$ have index invariants in trivial groups (since $[0,1]$ is contractible). But the element $\tau\left[A_{1}, A_{2}\right]=\tau[S] \in \mathcal{E x t}\left(S^{1}\right)$ is nonzero (corresponding to the well-known fact that the unilateral shift cannot be written as $S=B_{1}+i B_{2} \bmod \mathscr{K}$, with $B_{1}$ and $B_{2}$ commuting selfadjoints). Even if

$$
j t \sigma_{e}\left(A_{1}, A_{2}\right) \cong \sigma_{e}\left(A_{1}\right) \times \sigma_{e}\left(A_{2}\right)
$$

there may be nontrivial index invariants created, as it were, ex nihilo. The case of operators $A_{1}, A_{2}$ satisfying (6.18) is considerably easier to handle, however. It would be of interest to find sufficient conditions on $A_{1}, A_{2}$ to ensure (6.18). If the joint essential spectrum of the operators involved is quite simple then Ext can on occasion be computed. The case of essential projections (operators which are compact perturbations of projections) illustrates this point.

Corollary (6.19). Let $A_{1}, \ldots, A_{k}$ be essential projections, let $N$ be an essentially normal operator with $\sigma_{e}(N)=Y$ homeomorphic to a subset of $\mathbf{R}$ and suppose that the $C^{*}$-algebra generated by $\left\{I, \pi A_{1}, \ldots, \pi A_{k}, \pi N\right\}$ is commutative. (Denote it as $C(X)$.) Then there exist commuting normals $B_{1}, \ldots, B_{k}, C$ with $A_{j}=B_{j}+K_{j}$, (hence the $B_{j}$ are essential projections), $N+K=C$ and the $K_{j}$ and $K$ are compact.

Proof. The space $X$ is homeomorphic to a subspace of $Y \times\{0,1\}^{k}$ which embeds in $\mathbf{R}$. Thus so does $X$, and $\operatorname{Ext}(X)=0$. The extension $\tau\left[A_{1}, \ldots, A_{k}\right.$, $N]$ is thus zero.

Remark (6.20). The case of several almost commuting operators $A_{1}, \ldots, A_{n}$ proceeds as in the two-operator case, up to a point. The critical invariant is

$$
\tau\left[A_{1}, \ldots, A_{n}\right] \in \operatorname{Ext}(X)
$$

where $X=j t \sigma_{e}\left(A_{1}, \ldots, A_{n}\right) \subset R^{2 n}$.

But, lacking further information on $X$, there is no reason to suppose that $\mathcal{E x t}(X)$ is effectively computable. The whole connection between $A_{1}, \ldots, A_{n}$ and ${ }^{s} H_{*}(X)$ seems mysterious. For example, what conditions on the $A_{j}$ would ensure that ${ }^{s} \tilde{H}_{*}(X)=0$ ?

7. The Chern character and differentials in the spectral sequence. Suppose that $X$ is compact metric and $E^{2}=E^{\infty}$ in the spectral sequence converging to $\varepsilon_{*}(X)$. Then, modulo group extension difficulties, one has

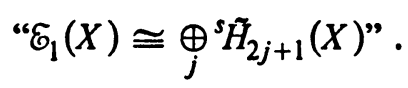


This suggests, first of all, a connection in general between $\delta x t(X)$ and $\oplus_{j}{ }^{s} \tilde{H}_{2 j+1}(X)$, and, second of all, the need to avoid group extension difficulties. The Chern character executes both of these ideas.

First some notation. If $A_{*}$ is a graded abelian group, let $A_{+}=\oplus_{n} A_{2 n}, A_{-}$ $=\oplus_{n} A_{2 n+1}$ and $A_{* *}=A_{+} \oplus A_{-}$regarded as a $\mathrm{Z} / 2$ graded group. In cohomology replace sums by products: $A^{+}=\prod_{n} A^{2 n}, A^{-}=\prod_{n} A^{2 n+1}$, and $A^{* *}$ $=A^{+} \oplus A^{-}=\Pi_{n} A^{n}$.

Proposition (7.1) (ATIYAH AND HiRZebruch) [8]. There is a natural transformation of $\mathbf{Z} / 2$ graded cohomology theories

$$
\text { ch: } K^{*}(X) \otimes \mathbf{Q} \rightarrow \check{H}^{* *}(X ; \mathbf{Q})
$$

called the Chern character, which is an isomorphism if $X$ is a finite complex.

In this section we define

$$
\operatorname{ch}: \varepsilon_{*}(X) \rightarrow \check{H}_{*}(X ; \mathbf{Q})={ }^{s} \tilde{H}_{*}(X ; \mathbf{Q}) .
$$

Then the map

$$
\operatorname{ch} \otimes \mathbf{Q}: \mathcal{E}_{*}(X) \otimes \mathbf{Q} \rightarrow \check{H}_{* *}(X ; \mathbf{Q})
$$

will be an isomorphism on finite complexes. (Recall that to simplify notation, we understand $\check{H}_{*}$ to be reduced Čech homology theory.)

Suppose $X$ is compact metric, with $X=$ proj $\lim X_{j}$, the inverse limit of finite complexes. Then

$$
\tilde{K}^{*}(X)=\operatorname{inj} \lim \tilde{K}^{*}\left(X_{j}\right) \text { and } \check{H}^{*}(X)=\operatorname{inj} \lim \tilde{H}^{*}\left(X_{j}\right)
$$

so there is a natural isomorphism ch, defined to be the composite

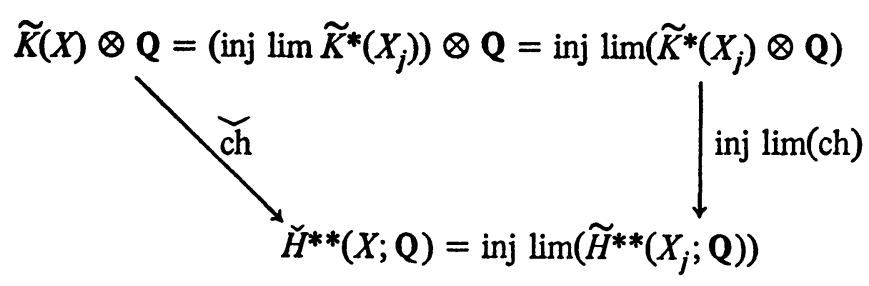

Now hom the diagram (7.3) into $\mathbf{Q}$ and observe that

$$
{ }^{s} \tilde{H}_{* *}(X ; \mathbf{Q})=\operatorname{hom}\left(\check{H}^{* *}(X ; \mathbf{Q}), \mathbf{Q}\right)
$$

via the UCT (0.4). Then (7.3) yields a natural isomorphism 


$$
\mathrm{ch}^{*}:{ }^{s} \tilde{H}_{* *}(X ; \mathbf{Q}) \rightarrow \operatorname{hom}\left(\tilde{K}^{*}(X) \otimes \mathbf{Q}, \mathbf{Q}\right) .
$$

Finally, define the Chern character for $\mathcal{E}_{*}$ to be the composite

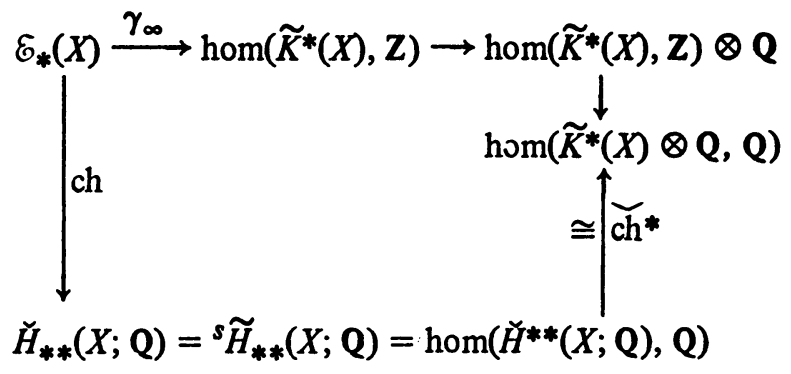

where $\gamma_{\infty}$ is the index map (5.3). When convenient, consider $\mathrm{ch}$ to be $\mathrm{ch}_{0} \oplus \mathrm{ch}_{1}$ where $\mathrm{ch}_{i}: \mathcal{E}_{i}(X) \rightarrow \check{H}_{ \pm}(X ; \mathbf{Q})$.

Obviously ch vanishes on the torsion subgroup of $\varepsilon_{*}(X)$. The precise identification of the kernel and cokernel of $c h \otimes \mathbf{Q}$ is of some interest.

THEOREM (7.7). Let $X$ be compact metric of finite dimension and let $\left\{E^{r}, d^{r}\right\}$ be the spectral sequence converging to $\mathcal{E}_{*}(X)$ with $E^{2}={ }^{s} \tilde{H}_{*}\left(X ; \mathcal{E}_{*}\left(S^{0}\right)\right)$. Then the following are equivalent:

(a) The differentials in $\left\{E^{r}, d^{r}\right\}$ are torsion-valued and $\operatorname{ch} \otimes \mathbf{Q}: \mathcal{E}_{*}(X) \otimes \mathbf{Q}$ $\rightarrow{ }^{s} \tilde{H}_{* *}(X ; \mathbf{Q})$ is an isomorphism.

(b) $\operatorname{hom}\left(\breve{H}_{*}(X), \mathbf{Q} / \mathbf{Z}\right) \otimes \mathbf{Q}=0$.

REMARK (7.8). To assert that differentials are torsion-valued means that the image of each $d^{r}$ (for $r \geqslant 2$ ) is contained in the torsion subgroup of $E^{r}$, or equivalently, that $d_{r} \otimes \mathbf{Q}=0$. Note that ${ }^{s} H_{*}(X ; \mathbf{Q}) \otimes \mathbf{Q}={ }^{s} H_{*}(X ; \mathbf{Q})$.

Let $G(A)=\operatorname{hom}(A, \mathbf{Q} / \mathbf{Z}) \otimes \mathrm{Q}$ for convenience.

Lemma (7.9). $G\left(\check{H}^{*}(X)\right)=0$ if and only if the natural map $\iota:{ }^{s} \tilde{H}_{*}(X) \otimes \mathbf{Q}$ $\rightarrow{ }^{s} \tilde{H}_{*}(X ; \mathbf{Q})$ is an isomorphism.

Proof. Consider the long exact coefficient sequence for Steenrod homology associated to $\mathbf{Z} \rightarrow \mathbf{Q} \rightarrow \mathbf{Q} / \mathbf{Z}$. Apply $\otimes \mathbf{Q}$ and it reads

$$
\begin{aligned}
\cdots & \rightarrow{ }^{s} \tilde{H}_{n+1}(X ; \mathbf{Q} / \mathbf{Z}) \otimes \mathbf{Q} \rightarrow{ }^{s} \tilde{H}_{n}(X) \otimes \mathbf{Q} \rightarrow{ }^{s} \tilde{H}_{n}(X ; \mathbf{Q}) \\
& \rightarrow{ }^{s} \tilde{H}_{n}(X ; \mathbf{Q} / \mathbf{Z}) \otimes \mathbf{Q} ;
\end{aligned}
$$

hence $\iota$ is an isomorphism exactly when ${ }^{s} \tilde{H}_{*}(X ; \mathbf{Q} / \mathbf{Z}) \otimes \mathbf{Q}=0$. But the UCT implies that ${ }^{s} \tilde{H}_{*}(X ; \mathbf{Q} / \mathbf{Z})=\operatorname{hom}\left(\check{H}^{*}(X), \mathbf{Q} / \mathbf{Z}\right)$ (since $\mathbf{Q} / \mathbf{Z}$ is divisible) and hence

$$
{ }^{s} \tilde{H}_{*}(X ; \mathbf{Q} / \mathbf{Z}) \otimes \mathbf{Q}=G\left(\check{H}^{*}(X)\right) \text {. }
$$


Now to the proof of Theorem (7.7), which is essentially the same as AtiyahHirzebruch [8]. Let $E_{\mathbf{Q}}^{r}=E^{r} \otimes \mathbf{Q}$. This is a $\mathbf{Z} / 2$ graded spectral sequence converging to $\mathscr{E}_{*}(X) \otimes \mathbf{Q}$, with $E_{\mathbf{Q}}^{2}={ }^{s} \tilde{H}_{*}\left(X ; \mathcal{E}_{*}\left(S^{0}\right)\right) \otimes \mathbf{Q}$. Thus statement (a) is equivalent to

$\left(a^{\prime}\right) E_{\mathbf{Q}}^{2}=E_{\mathbf{Q}}^{\infty}$ and $\operatorname{ch} \otimes \mathbf{Q}$ is an isomorphism.

Let $E_{T}^{Y}$ ( $T$ for "trivial") be the $\mathbf{Z} / 2$ graded spectral sequence obtained by regarding $\breve{H}_{* *}(X ; \mathbf{Q})$ as a generalized Steenrod homology theory. Then $E_{T}^{2}=E_{T}^{\infty}=\breve{H}_{* *}(X ; \mathbf{Q})$. Since ch $\otimes \mathbf{Q}$ is natural, it induces a morphism of spectral sequences $(3.5)$

$$
\tau_{r}: E_{\mathbf{Q}}^{r} \rightarrow E_{T}^{r}
$$

such that $\tau_{2}=\iota$ and $\tau_{\infty}$ is the associated graded map to ch $\otimes \mathbf{Q}$. Thus (b) is equivalent to

$\left(b^{\prime}\right) \tau_{2}$ is an isomorphism by Lemma (7.9). It remains to show that $\left(a^{\prime}\right)$ is equivalent to $\left(b^{\prime}\right)$.

Suppose $\left(\mathrm{a}^{\prime}\right)$ holds. Then $\tau_{\infty}$ is an isomorphism (since $\operatorname{ch} \otimes \mathbf{Q}$ is). But then one has

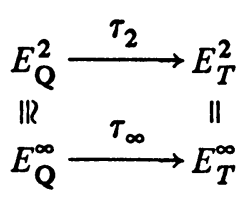

$\left(E_{\mathbf{Q}}^{2}=E_{\mathbf{Q}}^{\infty}\right.$ by assumption $\left.\left(a^{\prime}\right)\right)$, so $\tau_{2}$ is an isomorphism, proving $\left(b^{\prime}\right)$.

Conversely, suppose that $\left(b^{\prime}\right)$ holds; $\tau_{2}$ is an isomorphism. Then $\tau_{r}$ is an isomorphism for all $r \leqslant \infty$, the spectral sequence $E_{\mathbf{Q}}^{r}$ collapses, so $E_{\mathbf{Q}}^{2}=E_{\mathbf{Q}}^{\infty}$ and $\tau_{\infty}$ is an isomorphism. Now $\tau_{\infty}$ is the associated graded map to ch $\otimes \mathbf{Q}$. Then $c h \otimes \mathbf{Q}$ is clearly surjective and a direct argument shows it is injective, proving $\left(\mathrm{a}^{\prime}\right)$.

RemarK (7.10). If $X$ is a finite complex, then $G\left(\check{H}^{*}(X)\right)=0$. In fact, $G(A)=0$ if and only if $\operatorname{rank}_{\mathrm{Q}} A<\infty$ and $\operatorname{Ext}(A, \mathbf{Z})$ is a torsion group.

For an example of a space $X$ with $G\left(\check{H}^{*}(X)\right) \neq 0$, take the dyadic solenoid. Then $\check{H}^{1}(X)=\mathbf{Z}\left[\frac{1}{2}\right]$ and $G\left(\check{H}^{*}(X)\right)=\check{\mathbf{Q}}_{2}$, the 2-adic numbers.

It is possible to explicitly say when $\operatorname{ch} \otimes \mathbf{Q}$ is an isomorphism.

THEOREM (7.11). There is a natural short exact sequence

$$
0 \rightarrow \operatorname{cok}(\operatorname{ch} \otimes \mathbf{Q}) \rightarrow G\left(\tilde{K}^{*}(X)\right) \rightarrow \operatorname{ker}(\operatorname{ch} \otimes \mathbf{Q}) \rightarrow 0
$$

so $\mathrm{ch} \otimes \mathbf{Q}$ is an isomorphism if and only if $G\left(\tilde{K}^{*}(X)\right)=0$.

Proof. The map ch $\otimes \mathbf{Q}$ is the composite 


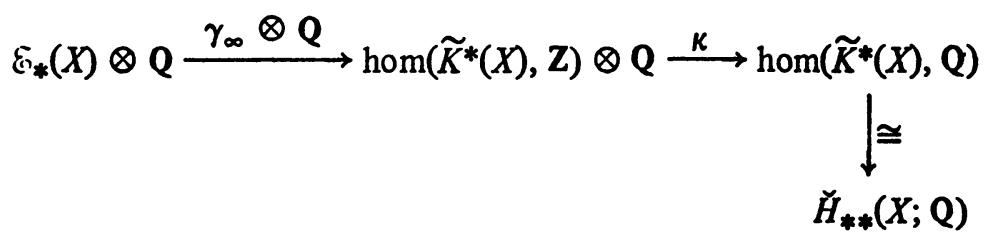

Since $\gamma_{\infty}$ is surjective and $\kappa$ is injective, it is immediate that

$$
\operatorname{ker}(\operatorname{ch} \otimes \mathbf{Q})=\operatorname{ker}\left(\psi_{\infty} \otimes \mathbf{Q}\right)=\operatorname{Ext}\left(\tilde{K}^{*}(X), \mathbf{Z}\right) \otimes \mathbf{Q}
$$

and

$$
\operatorname{cok}(\operatorname{ch} \otimes \mathbf{Q})=\operatorname{cok}(\kappa) .
$$

Applying $\operatorname{hom}\left(\tilde{K}^{*}(X),-\right) \otimes \mathbf{Q}$ to the sequence $0 \rightarrow \mathbf{Z} \rightarrow \mathbf{Q} \rightarrow \mathbf{Q} / \mathbf{Z} \rightarrow 0$ yields the long exact sequence

$$
\begin{gathered}
0 \rightarrow \operatorname{hom}\left(\widetilde{K}^{*}(X), \mathbf{Z}\right) \otimes Q \stackrel{\kappa}{\longrightarrow} \operatorname{hom}\left(\widetilde{K}^{*}(X), Q\right) \\
\stackrel{\beta}{\longrightarrow} G\left(\widetilde{K}^{*}(X)\right) \stackrel{\delta}{\longrightarrow} \operatorname{Ext}\left(\widetilde{K}^{*}(X), Z\right) \otimes Q \rightarrow 0 \\
\| \\
\operatorname{ker}(\operatorname{ch} \otimes Q)
\end{gathered}
$$

Thus $\operatorname{im} \delta=\operatorname{ker}(\operatorname{ch} \otimes Q)$. Further,

$$
\begin{aligned}
\operatorname{cok}(\operatorname{ch} \otimes \mathbf{Q}) & =\operatorname{cok}(\kappa)=\operatorname{hom}\left(\tilde{K}^{*}(X), \mathbf{Q}\right) / \operatorname{im}(\kappa) \\
& =\operatorname{hom}\left(\tilde{K}^{*}(X), \mathbf{Q}\right) / \operatorname{ker}(\beta) \\
& \cong \operatorname{im}(\beta)=\operatorname{ker}(\delta) .
\end{aligned}
$$

Then the natural commutative diagram

$$
\begin{aligned}
& 0 \longrightarrow \operatorname{ker}(\delta) \longrightarrow G\left(\widetilde{K}^{*}(X)\right) \longrightarrow \operatorname{im} \delta \longrightarrow 0 \\
& \mathbb{R} \quad \mathbb{} \quad \mathbb{R} \\
& 0 \rightarrow \operatorname{cok}(\operatorname{ch} \otimes Q) \rightarrow G\left(\widetilde{K}^{*}(X)\right) \rightarrow \operatorname{ker}(\operatorname{ch} \otimes Q) \rightarrow 0
\end{aligned}
$$

yields the theorem.

REMARK (7.12). The functor $G(A)=\operatorname{hom}(A, \mathbf{Q} / \mathbf{Z}) \otimes \mathbf{Q}$ spotlights a curious phenomenon. We have shown

(i) $G\left(\tilde{K}^{*}(X)\right)=0$ if and only if $\operatorname{ch} \otimes \mathbf{Q}$ is an isomorphism, and

(ii) $G\left(\check{H}^{*}(X)\right)=0$ if and only if $\operatorname{ch} \otimes \mathbf{Q}$ is an isomorphism and the 
differentials in the appropriate spectral sequence are torsion valued.

Thus $G\left(\breve{H}^{*}(X)\right)=0$ implies that $G\left(\tilde{K}^{*}(X)\right)=0$. Is the converse true?

8. The algebraic structure of $8 \mathrm{xt}(X)$. This section is devoted to coping with the problems which arise from the fact that $\delta x t(X)$ need not be a finitely generated abelian group.

Recall that if $X=$ proj $\lim X_{j}$ where the $X_{j}$ are finite complexes and $h_{*}$ is a Steenrod homology theory then we have defined

$$
\check{h}_{n}(X)=\operatorname{proj} \lim h_{n}\left(X_{j}\right) \text { and } e h_{n}(X)=\operatorname{proj} \lim ^{1} h_{n+1}\left(X_{j}\right)
$$

and have shown that these groups are independent of the CNS $X=\left\{X_{j}\right\}$. Let $H_{+}(X)=\oplus_{j} H_{2 j}(X)$ and $H_{-}(X)=\oplus_{j} H_{2 j+1}(X)$. Note that we may assume $\operatorname{dim} X \geqslant \operatorname{dim} X_{j}$ in calculating the above groups.

THEOREM (8.1). Let $X$ be compact metric of finite dimension. Suppose that $\mathcal{L} H_{*}(X)=0$. Then $\mathfrak{L E}_{*}(X)=0$ and $\operatorname{Ext}(X)=\check{K}_{1}(X)$.

Proof. We give the proof for $\mathfrak{L E}_{0}(X)$. The cohomology Chern character $\tilde{K}^{0}(X) \rightarrow \check{H}^{+}(X ; Q)$ induces a natural transformation $c: \tilde{K}^{0}(X) / t \tilde{K}^{0}(X)$ $\rightarrow H^{+}(X ; Q)$ where $t A$ is the torsion subgroup of $A$. The image of $c$ may not be integral (i.e. it may not lie in the image of $H^{+}(X) \rightarrow H^{+}(X ; Q)$ ). However Adams [1] shows there is an integer $\mu(i)$ such that $c_{i}=\mu(i) \cdot c$ is integral for all finite complexes $Y$ of dimension $\leqslant i$. The map

$$
c_{i}: \tilde{K}^{0}(Y) / t \tilde{K}^{0}(Y) \rightarrow H^{+}(Y) / t H^{+}(Y)
$$

is a natural injection, and $\varphi(Y)=\operatorname{coker}\left(c_{i}\right)$ is a finite group.

The sequence

$$
\begin{array}{r}
0 \rightarrow \operatorname{hom}\left(H^{+}\left(X_{j}\right) / t H^{+}\left(X_{j}\right), \mathbf{Z}\right) \rightarrow \operatorname{hom}\left(\widetilde{K}^{0}\left(X_{j}\right) / t \widetilde{K}^{0}\left(X_{j}\right), \mathbf{Z}\right) \\
\downarrow \\
\operatorname{Ext}\left(\varphi\left(X_{j}\right), \mathrm{Z}\right) \rightarrow 0
\end{array}
$$

is thus exact, for each $j$. Take inverse limits over $j$ to obtain

$$
\begin{aligned}
& \text { proj } \lim ^{1} \operatorname{Ext}\left(\varphi\left(X_{j}\right), \mathbf{Z}\right) \longleftarrow \operatorname{proj} \lim ^{1} \operatorname{hom}\left(\widetilde{K}^{0}\left(X_{j}\right) / t \widetilde{K}^{0}\left(X_{j}\right), \mathbf{Z}\right) \\
& \text { II } \\
& 0 \\
& \text { proj } \lim ^{1} \operatorname{hom}\left(H^{+}\left(X_{j}\right) / t H^{+}\left(X_{j}\right), \mathbf{Z}\right)
\end{aligned}
$$

Then Theorem (8.1) follows from this lemma. 
LeMma (8.2). Let $h^{*}=\tilde{K}^{0}$ or $H^{+}$and $h_{*}=\mathcal{E}_{0}$ or ${ }^{s} \tilde{H}_{+}$. Then

$$
\mathcal{L} h_{*}(X)=\operatorname{proj} \lim ^{1} \operatorname{hom}\left(h^{*}\left(X_{j}\right) / t h^{*}\left(X_{j}\right), \mathbf{Z}\right) .
$$

Proof. Both $\tilde{K}^{0}$ and $H^{+}$satisfy the UCT. Thus the natural map

$$
h_{*}\left(X_{j}\right) / t h_{*}\left(X_{j}\right) \rightarrow \operatorname{hom}\left(h^{*}\left(X_{j}\right), \mathbf{Z}\right) / t \operatorname{hom}\left(h^{*}\left(X_{j}\right), \mathbf{Z}\right)
$$

is an isomorphism. But

$$
\operatorname{hom}(A, \mathbf{Z}) / t \operatorname{hom}(A, \mathbf{Z}) \cong \operatorname{hom}(A / t A, \mathbf{Z})
$$

for finiteiy generated abelian groups $A$. Thus

$$
h_{*}\left(X_{j}\right) / t h_{*}\left(X_{j}\right) \cong \operatorname{hom}\left(h^{*}\left(X_{j}\right) / t h^{*}\left(X_{j}\right), \mathbf{Z}\right)
$$

for each $j$. Take limits; then

$$
\begin{aligned}
\mathcal{L} h_{*}\left(X_{j}\right) & =\operatorname{proj} \lim ^{1}\left(h_{*}\left(X_{j}\right) / t h_{*}\left(X_{j}\right)\right) \\
& =\operatorname{proj} \lim ^{1} \operatorname{hom}\left(h^{*}\left(X_{j}\right) / t h^{*}\left(X_{j}\right), \mathbf{Z}\right)
\end{aligned}
$$

proving the lemma.

Recall that the generalized index map $\gamma_{\infty}: \delta_{1}(X) \rightarrow$ hom $\left(\tilde{K}^{1}(X), Z\right)$ fits into the UCT (0.5). In the following theorem $\operatorname{ker}\left(\psi_{\infty}\right)$ is decomposed into profinite and divisible subgroups.

THEOREM (8.3). The sequence

$$
\begin{gathered}
0 \rightarrow E_{\|}(X) \rightarrow \operatorname{Ext}\left(K^{0}(X), Z\right) \rightarrow \operatorname{proj} \lim \left(t \widetilde{K}_{1}\left(X_{j}\right)\right) \rightarrow 0 \\
\operatorname{ker}\left(\gamma_{\infty}\right)
\end{gathered}
$$

is exact and natural for compact metric $X=$ proj $\lim X_{j}$. In particular, if $X$ is movable then

$$
\operatorname{ker}\left(\psi_{\infty}\right) \cong \operatorname{proj} \lim \left(t \tilde{K}_{1}\left(X_{j}\right)\right)
$$

which is profinite.

Proof. For each $j$ the sequence

$$
0 \rightarrow \operatorname{Ext}\left(\tilde{K}^{0}\left(X_{j}\right), \mathbf{Z}\right) \rightarrow \tilde{K}_{1}\left(X_{j}\right) \rightarrow \operatorname{hom}\left(\tilde{K}^{1}\left(X_{j}\right), \mathbf{Z}\right) \rightarrow 0
$$

is exact, by the UCT. Taking limits yields 


$$
\begin{aligned}
0 \rightarrow \text { proj } \lim \operatorname{Ext}\left(\widetilde{K}^{0}\left(X_{j}\right), Z\right) \rightarrow \check{K}_{1}(X) \rightarrow & \operatorname{hom}\left(\widetilde{K}^{1}(X), Z\right) \\
& \downarrow \\
0 & =\operatorname{proj} \lim ^{1} \operatorname{Ext}\left(\widetilde{K}^{0}\left(X_{j}\right), Z\right)
\end{aligned}
$$

and hence the following diagram has exact rows and columns.

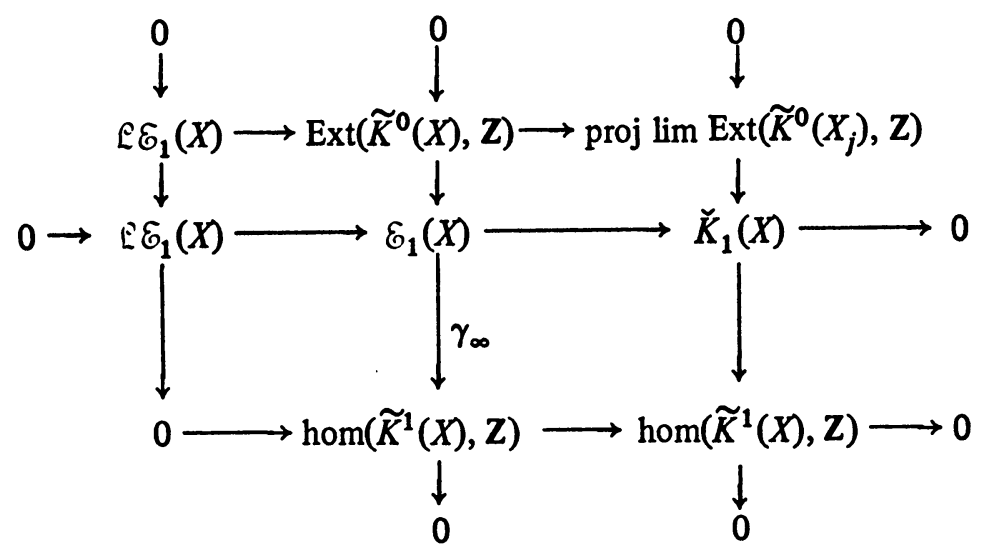

The serpent lemma (with $\gamma_{\infty}$ as the second map) yields the exact sequence

$$
\begin{aligned}
& 0 \rightarrow \mathfrak{L} \mathscr{E}_{1}(X) \rightarrow \operatorname{Ext}\left(K^{0}(X), \mathbf{Z}\right) \rightarrow \operatorname{proj} \lim \operatorname{ext}\left(\tilde{K}^{0}\left(X_{j}\right), \mathbf{Z}\right) \rightarrow 0 \\
& \operatorname{proj} \lim \left(t \tilde{K}_{1}\left(X_{j}\right)\right)
\end{aligned}
$$

proving the theorem.

REMARK (8.5). If $\mathrm{X}$ is a movable compact metric space then $\operatorname{ker}\left(\mathrm{ch}_{1}\right)$ $=$ proj $\lim \left(t \tilde{K}_{1}\left(X_{j}\right)\right)$, a profinite group. The strong wedge of a countable number of projective planes is a movable space for which $\mathrm{ch}_{1}$ is not injective.

The following theorem exhibits how $\mathfrak{L}_{1}(X)$ sits in $\operatorname{Ext}\left(\tilde{K}^{0}(X), Z\right)$ $=\operatorname{ker}\left(\nu_{\infty}\right)$.

THEOREM (8.6). Let $X$ be compact metric. Then $\mathfrak{L} \mathcal{F}_{1}(X)$ is the maximal divisible subgroup of $\delta \mathrm{xt}(X)$.

Proof. In the sequence (8.3), every divisible subgroup of $\operatorname{ext}\left(\tilde{K}^{0}(X), \mathbf{Z}\right)$ maps to zero. Thus $\mathfrak{L E}_{1}(X)$ contains all divisible groups and, being divisible itself, is the maximal divisible subgroup of $\operatorname{ker}\left(\chi_{\infty}\right)$ and hence also of $\operatorname{Ext}(X)$ (since im $\left(\psi_{\infty}\right)$ is reduced).

REMARK (8.7). It follows from Fuchs [23] that (8.6) is equivalent to each of the following statements:

(i) $\mathfrak{L E}_{1}(X)=P \operatorname{Ext}\left(\tilde{K}^{0}(X), Z\right)$, the pure exact sequences. 
(ii)

$$
\mathfrak{L} \mathscr{E}_{1}(X)=\cap_{k} k \operatorname{ext}\left(\tilde{K}^{0}(X), \mathbf{Z}\right),
$$

the first Ulm subgroup of $\operatorname{Ext}\left(\tilde{K}^{0}(X), \mathbf{Z}\right)$.

REMARK (8.8). We have shown that

$$
\operatorname{ker}(\mathrm{ch}) \cong\left\llcorner\mathscr{F}_{1}(X) \oplus \operatorname{proj} \lim \left(t \tilde{K}_{1}\left(X_{j}\right)\right) .\right.
$$

It would seem, in light of (8.9), that there are two sorts of phantom map behavior for $\mathscr{E}_{*}$. The first, corresponding to $\mathcal{L} \mathcal{E}_{*}$, is familiar; Proposition (2.15) gives a geometric interpretation analogous to the $K^{*}$ phantom classes. The second, corresponding to proj $\lim t \tilde{K}_{*}\left(X_{j}\right)$, consists of extensions which are of finite order when extended to any neighborhood of $X$ in the Hilbert cube, yet are not themselves of finite order.

Note that the groups $G\left(\tilde{K}^{*}(X)\right.$ ) (whose vanishing guarantees that $\operatorname{ch} \otimes \mathbf{Q}$ is an isomorphism) and $\operatorname{Ext}\left(\tilde{K}^{*}(X), \mathbf{Z}\right) \otimes \mathbf{Q}$ (the kernel of ch $\otimes \mathbf{Q}$ ) are related by a short exact sequence

$$
0 \rightarrow(\Pi(\mathbf{Q} / \mathbf{Z})) \otimes \mathbf{Q} \rightarrow G\left(\tilde{K}^{*}(X)\right) \rightarrow \operatorname{Ext}\left(\tilde{K}^{*}(X), \mathbf{Z}\right) \otimes \mathbf{Q} \rightarrow 0
$$

where the number of copies of $\mathbf{Q} / \mathbf{Z}$ equals the rank of $\tilde{K}^{*}(X)$.

THEOREM (8.10). Let $X$ be compact metric. Then $\delta \mathrm{xt}(X)$ is (unnaturally) isomorphic to the following sum:

$$
\mathcal{E x t}(X)=\mathcal{L} \mathscr{E}_{1}(X) \oplus \operatorname{proj} \lim \left(t \tilde{K}_{1}\left(X_{j}\right)\right) \oplus \operatorname{hom}\left(\tilde{K}^{1}(X), \mathbf{Z}\right) .
$$

The first summand $\mathfrak{L E}_{1}(X)$ is divisible; the second is profinite.

Proof. Immediate from (8.9) and the UCT (0.5).

This theorem indicates the algebraic possibilities for the group $\varepsilon x t(X)$ in the same sense that Nunke-Rotman [41] does for singular cohomology.

REMARK (8.11). We close by observing that Milnor's lim $^{1}$ sequence $(0.3)$ and L. G. Brown's UCT (0.5) carry essentially the same information. In light of (8.10), the $\lim ^{1}$ sequence may be written

$$
0 \rightarrow \mathfrak{L} \mathcal{E}_{1}(X) \rightarrow \mathcal{E}_{1}(X) \rightarrow \operatorname{proj} \lim \left(t \tilde{K}_{1}\left(X_{j}\right)\right) \oplus \operatorname{hom}\left(\tilde{K}^{1}(X), \mathbf{z}\right) \rightarrow 0
$$

while the UCT may be written

(8.13) $0 \rightarrow \mathfrak{L} \mathcal{E}_{1}(X) \oplus \operatorname{proj} \lim \left(t \tilde{K}_{1}\left(X_{j}\right)\right) \rightarrow \mathcal{E}_{1}(X) \stackrel{\gamma_{\infty}}{\longrightarrow} \operatorname{hom}\left(\tilde{K}^{1}(X), \mathrm{Z}\right) \rightarrow 0$

and both sequences split unnaturally. Thus the sequences differ only as to where the profinite group proj $\lim \left(t \tilde{K}_{1}\left(X_{j}\right)\right)$ sits-in $\check{K}_{1}(X)$ or in $\operatorname{ker}\left(\psi_{\infty}\right)$. 


\section{REFERENCES}

1. J. F. Adams, On Chern characters and the structure of the unitary group, Proc. Cambridge Philos. Soc. 57 (1961), 189-199. MR 22 \# 12525.

2. - Stable homotopy and generalized homology, Univ. of Chicago Press, Chicago, Ill., 1974.

3. D. W. Anderson, Universal coefficient theorems for K-theory (mimeo).

4. M. F. Atiyah, Characters and cohomology of finite groups, Inst. Hautes Etudes Sci. Publ. Math. No. 9 (1961), 23-64. MR 26 \#6228.

5. Algebraic topology and elliptic operators, Comm. Pure Appl. Math. 20 (1967), 237-249. MR 35 \#2299.

6. - K-theory, Benjamin, New York and Amsterdam, 1967. MR 36 \#130.

7. Global theory of elliptic operators, Proc. Internat. Conf. on Functional Analysis and

Related Topics (Tokyo, 1969), Univ. of Tokyo Press, Tokyo, 1970, pp. 21-30. MR 42 \#1154.

8. M. F. Atiyah and F. Hirzebruch, Vector bundles and homogeneous spaces, Proc. Sympos.

Pure Math., vol. 3, Amer. Math. Soc., Providence, R.I., 1961, pp. 7-38. MR 25 \#2617.

9. - Analytic cycles on complex manifolds, Topology 1 (1962), 25-45. MR 26 \#3091.

10. H. Behncke and H. Leptin, Classification of $C^{*}$-algebras with a finite dual, J. Functional Analysis 16 (1974), 241-257.

11. A. Borel and J. C. Moore, Homology theory for locally compact spaces, Michigan Math. J. 7 (1960), 137-159. MR 24 \#A1123.

12. K. Borsuk, Notes on the theory of shape, Aarhus Univ., 1971 (mimeo).

13. L. G. Brown, The determinant invariant for operators with compact self-commutators, Proc. Conf. on Operator Theory, Lecture Notes in Math., vol. 345, Springer-Verlag, New York, 1973.

14. - Operator algebras and algebraic $K$-theory (to appear).

15. L. G. Brown, R. G. Douglas and P. A. Fillmore, Extensions of $C^{*}$-algebras, operators with compact self-commutators, and K-homology, Bull. Amer. Math. Soc. 79 (1973), 973-978. MR 49 $\# 11265$.

16. - Unitary equivalence modulo the compact operators and extensions of $C^{*}$-algebras, Proc. Conf. on Operator Theory, Lecture Notes in Math., vol. 345, Springer-Verlag, New York, 1973.

17. J. A. Deddens and J. G. Stampfli, On a question of Douglas and Fillmore, Bull. Amer. Math. Soc. 79 (1973), 327-330. MR 47 \#7483.

18. R. G. Douglas, Banach algebra techniques in the theory of Toeplitz operators, CBMS Regional Conference, Univ. of Georgia, 1972.

19. E. Dyer, Cohomology theories, Math. Lecture Note Series, Benjamin, New York and Amsterdam, 1969. MR 42 \#3780.

20. S. Eilenberg and S. MacLane, Group extensions and homology, Ann. of Math. (2) 43 (1942), 757-831. MR 4, 88.

21. S. Eilenberg and N. Steenrod, Foundations of algebraic topology, Princeton Univ. Press, Princeton, N.J., 1952. MR 14, 398.

22. K. E. Ekman, Indices on $C^{*}$-algebras through representations in the Calkin algebra, Duke Math. J. 41 (1974), 413-432. MR 50 \#2925.

23. L. Fuchs, Infinite abelian groups. Vol. I, Pure and Appl. Math., vol. 36, Academic Press, New York and London, 1970. MR 41 \#333.

24. B. I. Gray, Spaces of the same n-type, for all $n$, Topology 5 (1966), 241-243. MR 33 \#4929.

25. J. W. Helton and R. E. Howe, Integral operators: commutators, traces, index and homology,

Proc. Conf. on Operator Theory, Lecture Notes in Math., vol. 345, Springer-Verlag, New York, 1973.

26. L. Hodgkin, On the K-theory of Lie groups, Topology 6 (1967), 1-36. MR 35 \#4950.

27. D. Husemoller, Fibre bundles, McGraw-Hill, New York, 1966. MR 37 \#4821. 
28. K. Jänich, Vektorraumbündel und der Raum der Fredholm-Operatoren, Math. Ann. 161 (1965), 129-142. MR 32 \#8356.

29. C. U. Jensen, Les foncteurs dérivés de lim et leur applications en theorie des modules, Lecture Notes in Math., vol. 254, Springer-Verlag, New York, 1972.

30. D. S. Kahn, An example in Čech cohomology, Proc. Amer. Math. Soc. 16 (1965), 584. MR 31 \#4027.

31. J. Kaminker and C. Schochet, Ext $(X)$ from a homological point of view, Proc. Conf. on Operator Theory, Lecture Notes in Math., vol. 345, Springer-Verlag, New York, 1973.

32. C. N. Lee and F. Raymond, Cech extensions of contravariant functors, Trans. Amer. Math. Soc. 133 (1968), 415-434. MR 38 \#2767.

33. S. Lefschetz, Topology, Chelsea, New York, 1956.

34. S. Mardešić, n-dimensional $L C^{n-1}$ compacta are movable, Bull. Acad. Polon. Sci. Sér. Sci. Math. Astronom. Phys. 19 (1971), 505-509. MR 46 \#869.

35. - A survey of the shape theory of compacta, General Topology and its Relations to Modern Analysis and Algebra III (Proc. Third Prague Topological Sympos., 1971), Academic Press, New York, 1973, pp. 291-300.

36. S. Mardešić and J. Segal, Movable compacta and ANR-systems, Bull. Acad. Polon. Sci. Sér. Sci. Math. Astronom. Phys. 18 (1970), 649-654. MR 44 \# 1026.

37. - Shapes of compacta and ANR systems, Fund. Math. 72 (1971), 41-59. MR 45 \#7686.

38. W. S. Massey, Exact couples in algebraic topology. I-V, Ann. of Math. (2) 56 (1952), 363-396; ibid. (2) (1953), 248-286. MR 14, 672, 1111.

39. J. Milnor, On the Steenrod homology theory, Berkeley, 1961 (mimeo).

40. J. Milnor and M. Barratt, An example of anomalous singular homology, Proc. Amer. Math. Soc. 13 (1962), 293-297. MR 25 \#566.

41. R. J. Nunke and J. J. Rotman, Singular cohomology groups, J. London Math. Soc. 37 (1962), 301-336. MR 25 \#3975.

42. J. Roos, Sur les functeurs dérivés de lim. Applications, C. R. Acad. Sci. Paris 252 (1961), 3702-3704. MR 24 \#A1938.

43. G. B. Segal, Equivariant K-theory, Inst. Hautes Études Sci. Publ. Math. No. 34 (1968), 129-151. MR 38 \#2769.

44. K. Sitnikov, The duality law for non-closed sets, Dokl. Akad. Nauk SSSR 81 (1951), 359-362. (Russian) MR 13, 860.

45. E. G. Skljarenko, Homology theory and the exactness axiom, Uspehi Mat. Nauk 24 (1969), no. 5 (149), 87-140 = Russian Math. Surveys 24 (1969), no. 5, 91-142. MR 41 \#7676.

46. E. H. Spanier, Function spaces and duality, Ann. of Math. (2) 70 (1959), 338-378. MR 21 \#6584.

47. - Algebraic topology, McGraw-Hill, New York, 1966. MR 35 \# 1007.

48. E. H. Spanier and J.H. C. Whitehead, Duality in homotopy theory, Mathematika 2 (1955), 56-80. MR 17, 563.

49. N. Steenrod, Regular cycles of compact metric spaces, Ann. of Math. (2) 41 (1940), 833-851. MR 2, 73.

50. — Regular cycles on compact metric spaces, Lectures in Topology, Univ. of Michigan Press, Ann Arbor, Mich., 1941, pp. 43-55. MR 3, 132.

51. Joseph L. Taylor, A counterexample in shape theory, Bull. Amer. Math. Soc. 81 (1975), 629-632.

52. U. Venugopalkrishna, Fredholm operators associated with strongly pseudoconvex domains in $(C)^{n}$, J. Functional Analysis 9 (1972), 349-373. MR 47 \# 4051.

53. G. W. Whitehead, Generalized homology theories, Trans. Amer. Math. Soc. 102 (1962), 227-283. MR 25 \#573.

54. Y. S. Yeh, Higher inverse limits and homology theories, Thesis, Princeton Univ., 1959.

55. R. G. Douglas, Extensions of $C^{*}$-algebras and $K$-homology (to appear). 
56. J. Kaminker and C. Schochet, Steenrod homology and operator algebras, Bull. Amer. Math. Soc. 81 (1975), 431-434.

57.

Department of Mathematics, Indiana University-PuRdue University at Indianapolis, INDIANAPOLIS, INDIANA 46205 (Current address of J. Kaminker)

Department of Mathematics, Indiana University, Bloomington, Indiana 47401

Current address (C. Schochet): Department of Mathematics, Wayne State University, Detroit, Michigan 48202 